\title{
Structural and mechanistic basis of the EMC-dependent biogenesis of distinct transmembrane clients
}

Lakshmi E. Miller-Vedam* 1,4,5,7, Bastian Bräuning*2 ${ }^{2}$ Katerina D. Popova* 3,5,7 Nicole T. Schirle Oakdale ${ }^{4,7,8}$, Jessica L. Bonnar ${ }^{5,7}$, Jesuraj Rajan Prabu ${ }^{2}$, Elizabeth A. Boydston ${ }^{7,12}$, Natalia Sevillano ${ }^{11,9}$, Matthew J. Shurtleff ${ }^{7,10}$, Robert M. Stroud ${ }^{4}$, Charles S. Craik ${ }^{11}$, Brenda A. Schulman ${ }^{\# 2}$, Adam Frost ${ }^{\#}$, Jonathan S. Weissman ${ }^{\#, 6,7}$

*Co-first authors contributed equally

\#Co-corresponding authors

1. Molecular, Cellular, and Computational Biophysics Graduate Program, University of California, San Francisco, California, USA

2. Department of Molecular Machines and Signaling, Max Planck Institute of Biochemistry, Martinsried, Germany

3. Biomedical Sciences Graduate Program, University of California, San Francisco, California, USA

4. Department of Biochemistry \& Biophysics, University of California, San Francisco, California, USA

5. Department of Biology, Whitehead Institute, MIT, Cambridge, Massachusetts, USA

6. Howard Hughes Medical Institute, USA

7. Department of Cellular and Molecular Pharmacology, University of California, San Francisco, California, USA

8. Current Address - Gilead Sciences, Foster City, California, USA

9. Current Address - FairJourney Biologics, Porto, Portugal

10. Current Address - Department of Bioengineering, Stanford University, Stanford, California, USA

11. Department of Pharmaceutical Chemistry, University of California, San Francisco, California, USA.

12. Current Address -Whitehead Institute, MIT, Cambridge, Massachusetts, USA

** Correspondence: schulman@biochem.mpg.de (B.A.S), Adam.Frost@ucsf.edu (A.F.), and weissman@wi.mit.edu (J.S.W.) 


\section{$\underline{\text { Abstract }}$}

Membrane protein biogenesis in the endoplasmic reticulum (ER) is complex and failureprone. The ER membrane protein complex (EMC), comprising eight conserved subunits, has emerged as a central player in this process. Yet, we have limited understanding of how EMC enables insertion and integrity of diverse clients, from tail-anchored to polytopic transmembrane proteins. Here, yeast and human EMC cryo-EM structures reveal conserved intricate assemblies and human-specific features associated with pathologies. Structure-based functional studies revealed at least two separable EMC activities, as an insertase regulating tail-anchored protein levels and as a polytopic membrane protein holdase chaperone. These depend on mechanistically coupled yet spatially distinct regions including two lipidaccessible membrane cavities which confer client-specific regulation, and a novel, noninsertase EMC function mediated by the EMC lumenal domain. Our studies illuminate the structural and mechanistic basis of EMC's multifunctionality and point to its role in differentially regulating the biogenesis of distinct client protein classes.

\section{Introduction}

Integral membrane proteins serve diverse and critical cellular roles, including signal transduction, lipid biosynthesis, adhesion, and transport of molecules across the bilayer. In eukaryotic cells, the endoplasmic reticulum (ER) serves as the primary site of integral membrane protein synthesis, targeting (co- or post-translationally), insertion, folding and quality control (Ellgaard 2016; Costa, 2018). However, the features of membrane-spanning regions (e.g. low hydrophobicity, charged residues, non-optimal lengths, lipid- and ionbinding sites and hairpins or kinked transmembrane helices) that mediate important functions pose particular challenges for transmembrane protein biosynthesis and folding. Consequently, membrane protein biogenesis is prone to failure, and this can lead to cellular stress and disease (Marinko, 2019). Thus, it is important to understand the cellular factors that facilitate proper membrane protein biogenesis for such challenging clients.

The ER membrane protein complex (EMC) has emerged as a conserved player in the process of membrane protein biogenesis. It was first identified in Saccharomyces cerevisiae, as an abundant and stable multi-protein membrane complex whose disruption results in stress mirroring that caused by misfolded membrane proteins (Jonikas, 2009). Loss of the EMC in mammalian cells is associated with failed biogenesis and degradation of a subset of membrane proteins (Christianson, 2012). Accordingly, the EMC has been implicated in several mechanistically distinct steps of membrane protein biogenesis, stabilization, and quality control (Bircham, 2011; Richard, 2013; Satoh, 2015; Savidis, 2016; Shurtleff and Ithzak, 2018; Volkmar, 2018; Tian, 2019).

One well-established EMC function is as an insertase for terminal transmembrane helices. EMC's insertase function has been demonstrated for two classes of clients: low 
hydrophobicity tail-anchored proteins (i.e. those that contain C-terminal membrane anchors) and a subset of polytopic transmembrane proteins in which the first helix is inserted with the N-terminus in the lumen (Guna, 2018; Chitwood, 2018). However, many studies indicate EMC functions beyond initial insertion of $\mathrm{N}$ - or $\mathrm{C}$-terminal helices. The EMC has been implicated in the biogenesis and stability of many membrane protein classes that do not require a terminal transmembrane insertase (Bircham, 2011; Louie, 2012; Richard, 2013; Shurtleff and Ithzak, 2018; Coelho, 2019; Luo, 2002; Volkmar, 2018; Talbot, 2019; Petkovic, 2020). Recent studies have shown that the EMC is required for stability of internal transmembrane helices of human and viral multi-pass membrane proteins (Hiramatsu, 2019; Lin 2019; Ngo, 2019; Coelho, 2019; Xiong, 2020). Additionally, the human EMC (hEMC) physically interacts with the NS4A-B region of the Dengue Virus polyprotein following Sec61-dependent translocation and signal peptidase cleavage, suggesting roles in posttranslational stabilization of polytopic membrane proteins (Ngo, 2019; Lin 2019). Similarly, the $S$. cerevisiae EMC (yEMC) co-immunoprecipitated with full-length polytopic transmembrane clients, including Pmalp (Luo, 2002), Mrh1p, and Fks1p (Shurtleff and Ithzak, 2018). In addition to varying types of transmembrane protein clients, the EMC also associates with a range of regulatory factors, including many general and substrate-specific chaperones in the cytoplasm and in the ER lumen (Bagchi, 2016; Coelho, 2019; Kudze, 2018; Richard, 2013; Shurtleff and Ithzak, 2018).

The complex architecture of the EMC provides additional support for multifunctionality in membrane protein biogenesis. The EMC is an eight (yeast) or nine (mammalian) component, 248-284 kDa complex with considerable mass in the ER lumen, membrane and cytosol. The cytoplasmic domain contains conserved tetratricopeptide repeats (TPR) repeats in EMC2, and the human complex accommodates an additional subunit, EMC8/9, whose function is not yet understood. The ER lumenal domain in yeast does not contain an N-terminal EMC1 expansion seen in hEMC. Notably, the ER lumenal domain has been linked to a number of disease-associated phenotypes (Junes-Gill, 2010; Probert, 2015; Harel, 2016; Abu-Safieh, 2012; Diamantopoulou, 2017; Marquez, 2020), and presents the possibility of additional functions for the human lumenal domain. One EMC subunit (EMC3) shares limited sequence homology with a family of insertases that are evolutionarily related to the bacterial insertase YidC (Samuelson, 2000; Kumazaki, 2014; Borowska, 2015; Anghel, 2017), perhaps explaining the insertase function of the complex. Nonetheless, the elaboration of the EMC compared to other known transmembrane domain (TMD) insertases and a diverse client range suggest additional functionality that has so far eluded mechanistic explanation.

Here, we determined high-resolution cryo-EM structures of yeast and human EMC and characterized the phenotypes of three distinct classes of EMC clients associated with a series of structure-based EMC mutants. Both yEMC and hEMC structures reveal a path for transmembrane helix insertion from the cytoplasm into the membrane via a conserved cavity. Our structures and mutants also revealed a second lipid-filled cavity with regions of importance for all three client types probed. Analysis of human disease mutations in EMC1 and our structure-informed mutations enabled us to decouple the EMC insertase function from non-insertase functions and reveal a potential role of the EMC in differentially controlling the biogenesis of distinct classes of client proteins. These structure-function studies collectively 
establish that the EMC adopts a modular architecture enabling its diverse functions in membrane protein biogenesis.

\section{$\underline{\text { Results }}$}

\section{Overview of strategy to comprehensively reveal EMC structure and function}

To comprehensively dissect both conserved and species-specific functions of the EMC, we developed approaches to produce EMC for structure determination and broad mutational analysis (Figure 1). We developed systems to produce robust quantities of pure intact yEMC and hEMC to determine structures for the two organisms in which different facets of EMC function have been described in detail (Jonikas, 2009; Christianson, 2012; Guna, 2018; Shurtleff and Ithzak, 2018). Parallel efforts converged on an approach involving FLAG affinity-tagging of the EMC5 C-terminus, which was performed for endogenous yEMC and recombinant hEMC in human embryonic kidney (HEK) cells (Figure 1 - figure supplement 1-2).

In parallel, to enable testing of hypotheses based on structures, we created a suite of human (K562) knockout cell lines deleted for individual EMC subunits - EMC1 (lumen), EMC2 (cytoplasm), EMC3 and EMC5 (transmembrane) - and a series of reporters of EMCdependent transmembrane protein biogenesis (Figure 1 - figure supplement 3).

Reintroduction of the wildtype EMC subunits in the respective knockout cells fully rescued the knockout phenotype (Figure 1 - figure supplement 4-5). This allowed for introduction of structure-based mutations in EMC subunits into the respective knockout cells to determine features supporting biogenesis of fluorescently-tagged versions of three different types of EMC clients: the transmembrane domain of a C-terminal tail-anchored transmembrane protein (squalene synthase, SQS ${ }^{378-410}$ ) (Guna, 2018), a polytopic transmembrane protein that depends on the EMC N-terminal insertase activity (Beta 1 adrenergic receptor, B1AR) (Chitwood, 2018), and a polytopic transmembrane protein (Sigma intracellular receptor 2, TMEM97) whose biogenesis requires the Sec61 translocon but does require a terminal helix insertase (Figure 1 - figure supplement 3-7). Monitoring the effect of an EMC mutation on fluorescent reporter levels provided a quantitative measure of its impact on EMC-dependent biogenesis of each class of client protein. We subsequently mapped these mutations onto the structure grouped by reporter phenotype (Video 1). Western blots against the endogenous EMC subunits allowed us to control for mutational effects on the production and stability of the EMC complex itself (Figure 1 - figure supplement 4-5). This strategy thus distinguishes effects resulting from a global disruption of the EMC complex from those caused be specific disruption of one or more EMC functions. 


\section{FIGURE 1}

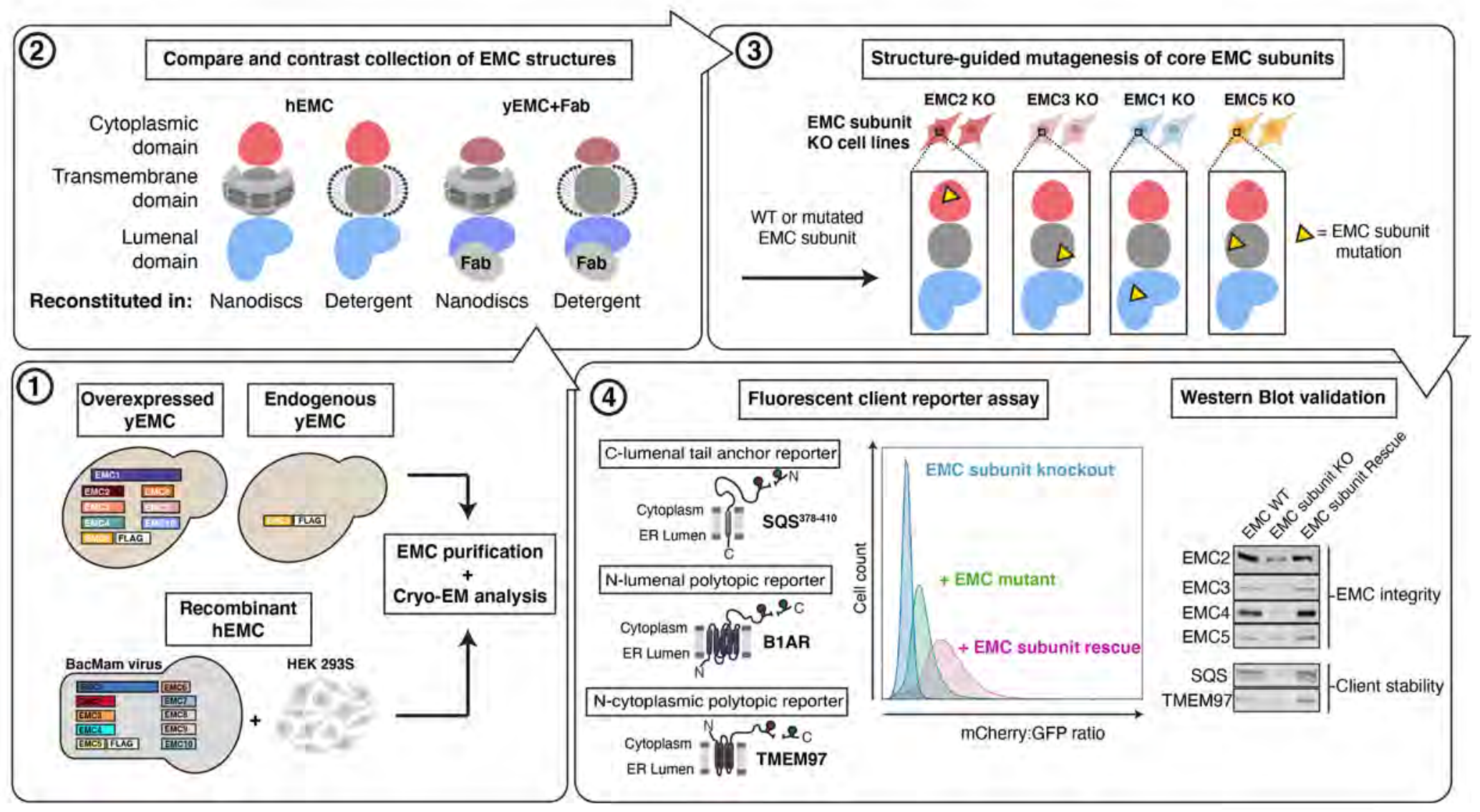

Figure 1: Experimental strategy for the dissection of EMC function.

Schematic representation of the combined structural and mutational approach to dissect EMC function.

(1) yEMC was expressed either by overexpression of all subunits together with Flag-tagged EMC5 or by endogenous expression from a genomic Flag-tagged EMC5 strain. For hEMC, all subunits were overexpressed together with Flag-tagged EMC5 via a single recombinant BacMam virus. EMC was purified by column chromatography and subjected to cryo-EM analysis.

(2) The obtained collection of cryo-EM structures of yEMC and hEMC in nanodiscs or detergent were compared to identify similarities and differences.

(3) Structure-guided mutagenesis was performed across four core EMC subunits: EMC1, EMC2, EMC3, and EMC5.

(4) Each EMC subunit knock-out (KO) cell line was individually transduced with three different fluorescent client reporters: SQS $378-410$, full length B1AR, and full length TMEM97. Mutant EMC subunits were introduced into KO cell lines missing the corresponding EMC subunit in each of the three fluorescent EMC client reporter cell lines. EMC client stability in each mutant EMC subunit cell line was assessed by fluorescent cell sorting and quantifying the mCherry-to-GFP ratio. Western blotting was performed for each mutant-transduced cell line to assess EMC integrity (by immunoblotting for core EMC subunits) as well as client stability (by immunoblotting for EMC clients) and compared against both wild-type (WT) and KO cell lines. 

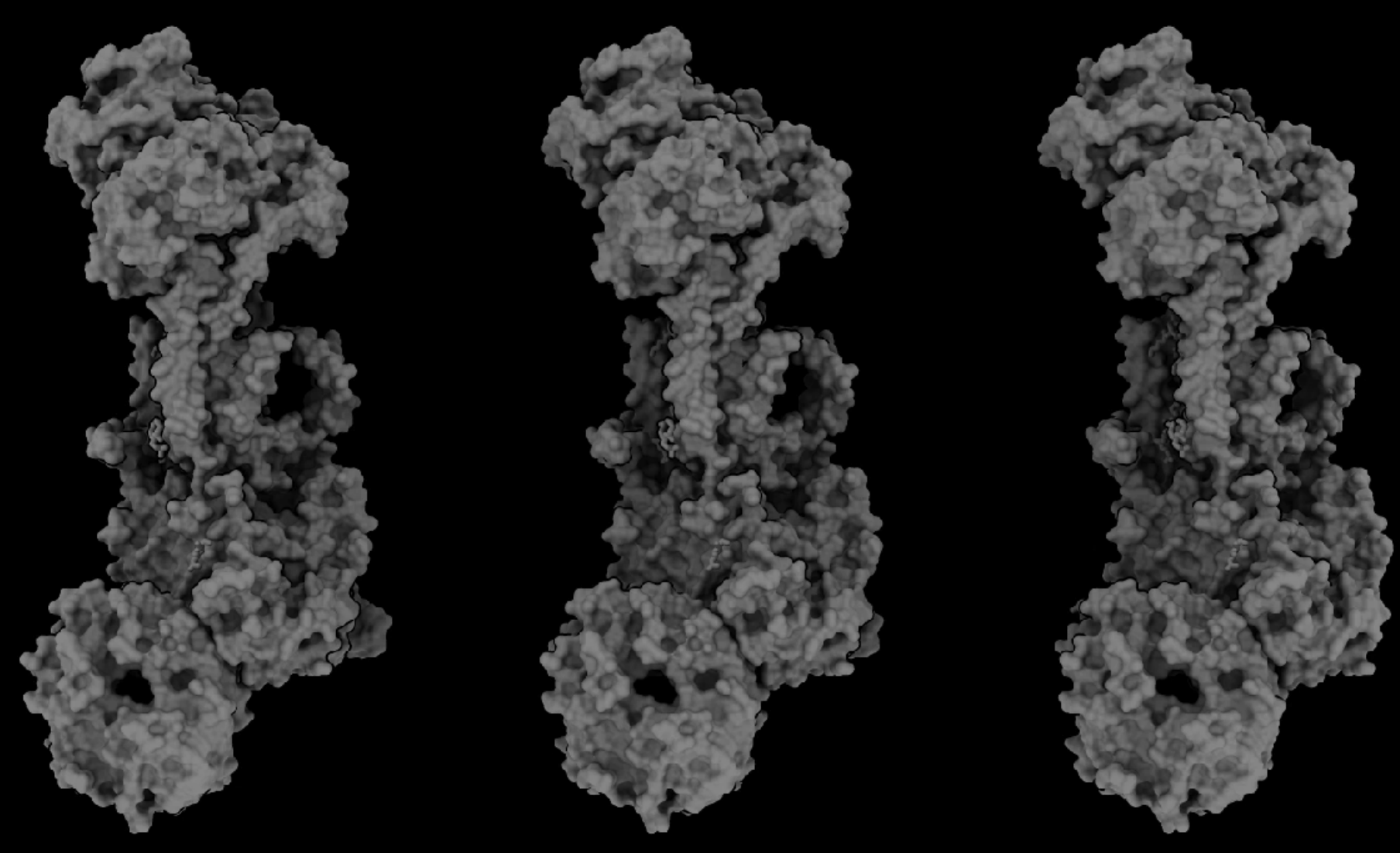

Video 1: hEMC mutagenesis displayed on hEMC structures

Three identical copies of hEMC in nanodisc are displayed here. Subsequent labeling and animation color mutated residues by flow cytometric measure of reporter abundance, grouped into three categories: increased reporter levels (mCherry $>$ GFP signal, colored magenta), wild type levels (mCherry signal is close to GFP signal, colored silver), and decreased reporter levels (mCherry $<$ GFP signal, colored gold). Left hEMC structure displays phenotypes for the C-lumenal tail anchor reporter (GFP-P2A-mCherry-SQS ${ }^{378-410}$ opsin). Middle hEMC structure displays phenotypes for the N-lumenal polytopic reporter (B1AR-mCherry-P2A-GFP). Right hEMC structure displays phenotypes for the Ncytoplasmic polytopic reporter (TMEM97-mCherry-P2A-GFP). Mild phenotypes are displayed as wild type levels 


\section{The EMC is an intricate molecular machine spanning the ER membrane and exhibits a conserved core architecture}

We determined structures of yEMC and hEMC - all showing overall compositional similarity, yet regional conformational differences between the yeast and human complexes (Figure 2). We obtained reconstructions of yEMC bound to an antigen binding fragment (Fab) and hEMC reconstituted both in detergent micelles and lipid nanodiscs, with the latter strategy yielding the most isotropic and highest resolution data. For yEMC+FabDH4 and hEMC, the global map resolutions reached 3.2 $\AA$ and $3.4 \AA$, respectively (Figure 2 - figure supplement 1 - 4). The cryo-EM maps allowed for de novo model building of both human and yeast complexes (Figure 2 - figure supplement 5, 6). We note that our maps and models are consistent with recent cryo-EM data from yeast EMC (Bai, 2020), human EMC (O’Donnell, Phillips, and Yagita, 2020; Pleiner, Tomaleri, and Januszyk, 2020), and a crystal structure of human EMC2-EMC9 (O'Donnell, Phillips, and Yagita, 2020) published while we were in the final stages of manuscript preparation. As described in the following sections, our multiple EMC structures enable a broad survey of its conserved architecture, with variations between the structures pointing to conformational and compositional differences (Figure 2 figure supplement 7).

The EMC comprises cytoplasmic, transmembrane and lumenal domains arranged similarly for yeast and human, despite significant evolutionary separation (Figure 2A-B). For both species, subunits encompassing EMC2 to EMC7 form an interconnected core complex, while there is additional density capping both the cytoplasmic and lumenal domains of hEMC, occupied by an EMC8/9 and an EMC1 N-terminal expansion, respectively (Figure 2C-D). EMC8 and EMC9 are paralogs of each other, which have not been identified in yeast (Wideman, 2015). We modeled and depict only EMC8 for clarity, but due to the $44 \%$ sequence identity with EMC9 and both being present in the recombinant system we refer to this as EMC8/9. The large EMC1 insertion in hEMC constitutes the majority of a membrane distal beta-propeller domain protruding into the lumen, a feature missing from $S$. cerevisiae. Compared to other ER-resident proteins (Suloway, 2009; Pfeffer, 2017; Ramírez, 2019), the arrangement of domains of the EMC is unusual with the transmembrane domain connecting prominent cytoplasmic and lumenal domains (Figure 2E). On a global level, the structure suggests complexities beyond those of some other ER machineries fulfilling select functions in transmembrane protein biogenesis. 


\section{FIGURE 2}
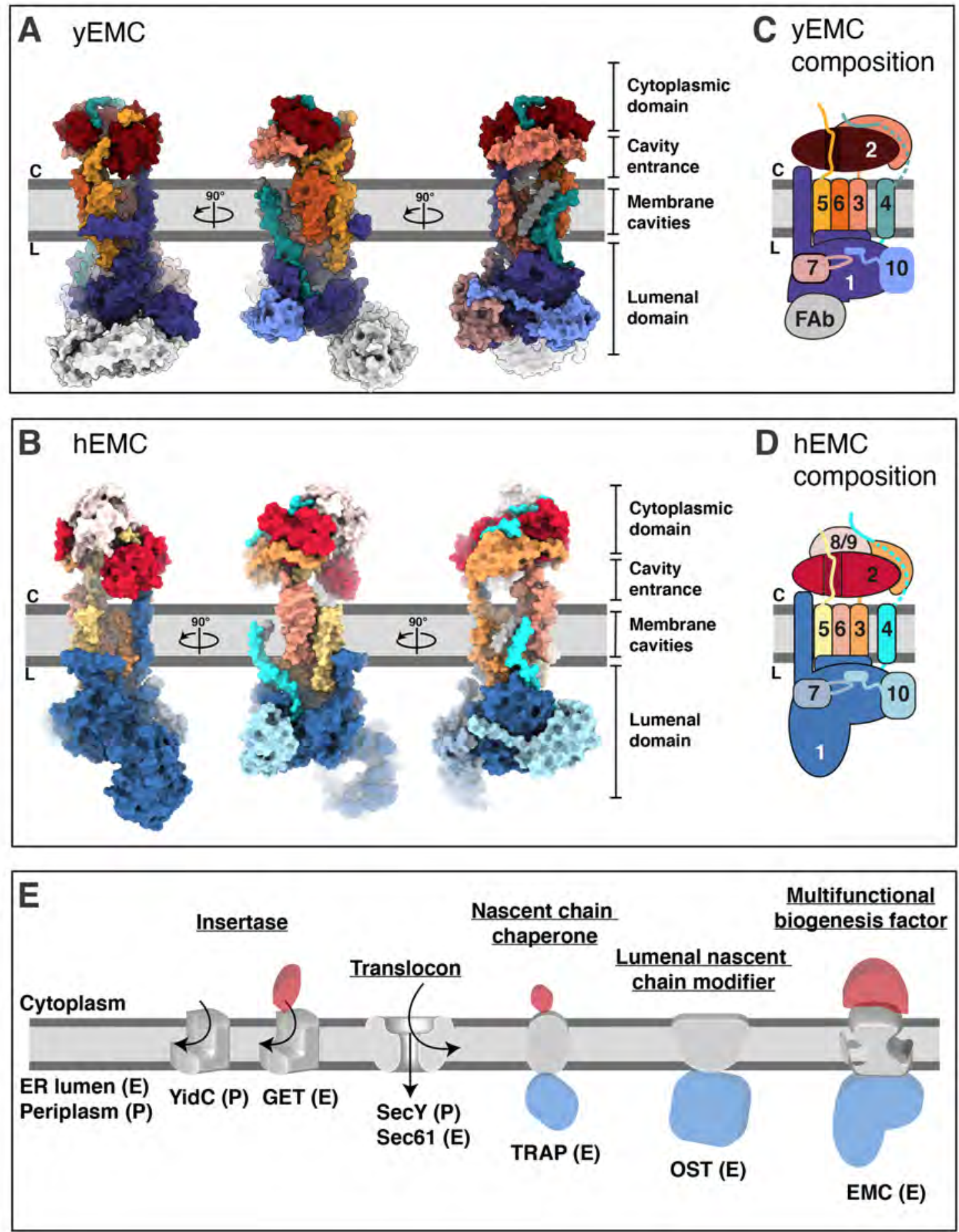

Figure 2: Overall structures of yeast and human EMC.

A) Cryo-EM structure of yEMC in nanodiscs. Three orthogonal views of the yEMC cryo-EM structure shown as surface rendering. Grey bars delineate the approximate ER membrane boundaries with the cytoplasmic $(\mathrm{C})$ and lumenal $(\mathrm{L})$ sides indicated. The FAb molecule bound to the $\mathrm{yEMC1}$ lumenal domain is colored in grey.

B) Cryo-EM structure of hEMC in nanodiscs. Labeling as in A).

C) Subunit composition and color scheme of yEMC used throughout the manuscript. Dotted line indicates a portion of EMC4 unresolved in the cryo-EM map and left unmodeled.

D) Subunit composition and color scheme of hEMC used throughout the manuscript.

E) Schematic depiction and comparison of the EMC architecture to known transmembrane protein biogenesis factors in the ER and the bacterial plasma membrane. Cytoplasmic, transmembrane and lumenal domains are depicted as cartoons colored red, grey and blue, respectively. E, eukaryotic; P, prokaryotic. 


\section{The cytoplasmic domain provides a platform for protein-protein interactions}

The exterior interface of the cytoplasmic domain is formed by EMC2, EMC3, EMC4, and parts of EMC8/9 (in human), while parts of EMC5, EMC2, and EMC8/9 are shielded from the cytoplasm (Figure 3A-B; Figure 2 - figure supplement 7). The helical fold of EMC2 constitutes the central organizer of this platform, established by five or six TPR motifs in human versus yeast, respectively (Figure 3C). TPR domains are commonly found mediating protein-protein interactions, and are present in numerous well-characterized chaperoneprotein and other interaction networks (Blatch, 1999; Scheufler, 2000; Schlegel, 2007; Assimon, 2015; Krysztofinska, 2017; Graham, 2019). Yeast EMC2 features a more curved helical arrangement with $\mathrm{N}$ - and C-terminal domains in closer proximity to each other than seen in human EMC2. Notably, the canonical peptide-binding TPR groove is occupied by the partially helical C-terminus of EMC5, which forms a large interaction surface with EMC2. To test the functional roles of this interaction, we mutated three residues within the EMC2 TPR motif (hEMC2 $\left.{ }^{\mathrm{K} 125 \mathrm{E}+\mathrm{R} 126 \mathrm{D}+\mathrm{K} 127 \mathrm{E}}\right)$ or a single EMC5 residue buried in the TPR binding groove $\left(\mathrm{hEMC} 5^{\mathrm{F} 90 \mathrm{~A}}\right)$. The mutations on both sides of the interface decreased EMC integrity, with a modest (for the single point mutation) and significant (for the multi-residue mutation) reduction in the levels of several EMC subunits (Figure 3C, Figure 3 - figure supplement 1-2, Figure 1 - figure supplement 4-5). This suggests that this interface might be critical for EMC complex assembly rather than activity (e.g. client binding).

The multi-protein cytoplasmic cap has distinct elements between hEMC and yEMC. Capping the cytoplasmic domain in hEMC is EMC8/9 (Figure 3 - figure supplement 3), the functional roles of this cap-like structure are not yet clear. A groove on EMC8/9 cradles an Nterminal peptide of EMC4, which proceeds into the EMC4 segment that traverses over EMC2 and the three-helix bundle of EMC3 (Figure 3D). Though yEMC lacks EMC8/9, yeast EMC4 follows a similar binding trajectory along cytoplasmic EMC2 and EMC3 surfaces. We mutated two patches of EMC2 residues in proximity to the EMC4 cytoplasmic region $\left(\mathrm{hEMC2} 2^{\mathrm{E} 146 \mathrm{~A}+\mathrm{E} 149 \mathrm{~A}+\mathrm{Q} 150 \mathrm{~A}}, \mathrm{hEMC2} 2^{\mathrm{E} 168 \mathrm{~A}+\mathrm{D} 170 \mathrm{~A}+\mathrm{K} 173 \mathrm{~A}}\right)$, which led to a modest accumulation of the tail-anchored client $\left(\mathrm{SQS}^{378-410}\right)$ but did not affect polytopic client abundance or decrease abundance of EMC subunits (Figure 3E-G, Figure 1 - figure supplement 4-5). Several mutants across the cytoplasmic domain showed similar phenotypes, supporting a key role in tail anchor protein biogenesis (Figure 3 - figure supplement 1-2). 


\section{FIGURE 3}
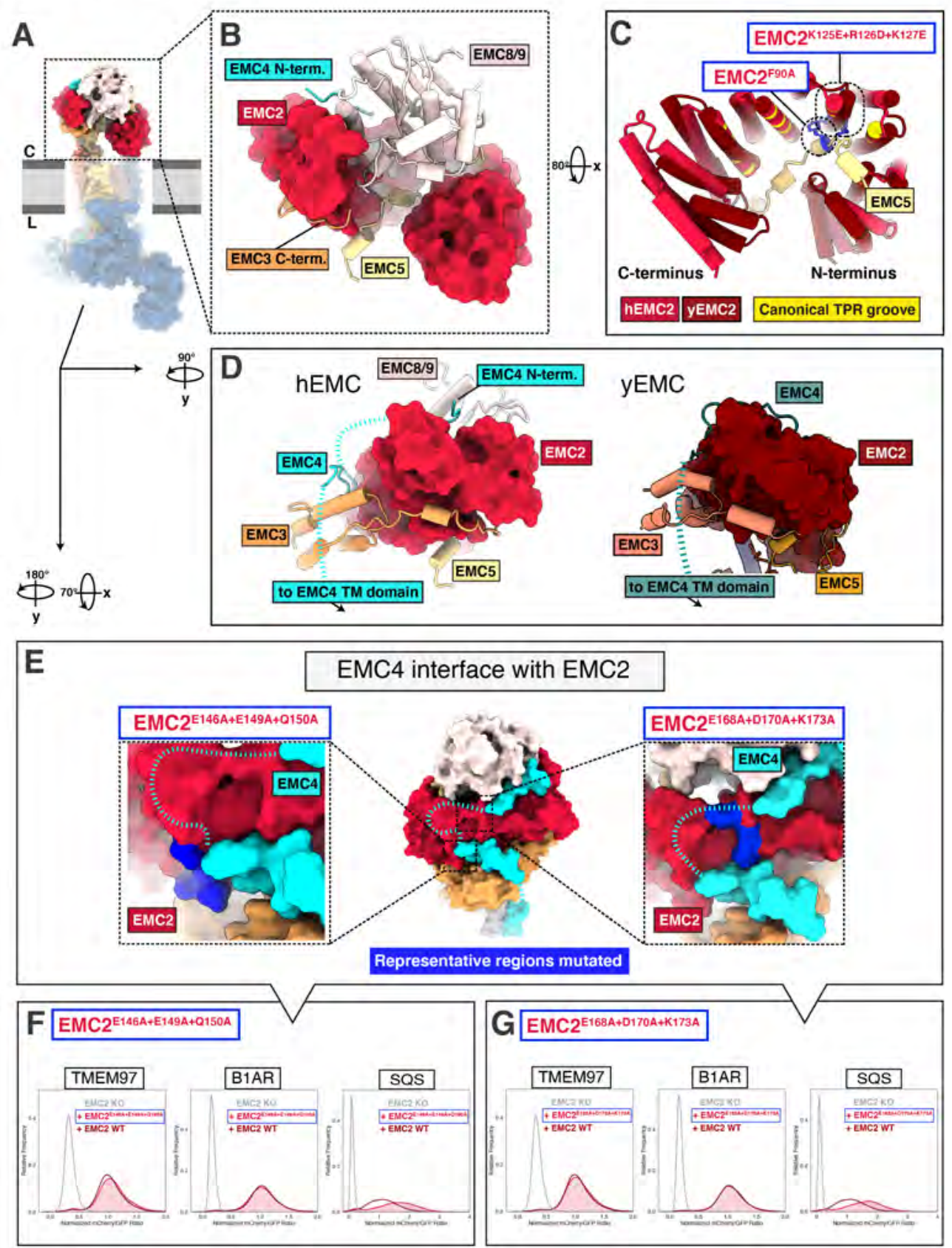

Figure 3: The EMC cytoplasmic domain contains conserved functional interfaces and may engage C-tail anchored clients directly.

A) Position of the hEMC cytoplasmic domain relative to the membrane and the rest of the complex. Shown is the surface rendered hEMC structure reconstituted in nanodiscs.

B) EMC2 nucleates a protein-protein interaction hub in the cytoplasm. Zoomed-in view of the cytoplasmic domain from A). EMC2 is shown as surface rendering while interacting EMC subunits are shown as cartoon cylinders.

C) EMC2 forms a TPR domain which binds EMC5. Overlaid are hEMC2 (red) and yEMC2 (dark red), illustrating the more tightly wound yEMC2 TPR solenoid. Canonical residues lining the concave TPR interior are colored in yellow stripes on the dark red for hEMC2. Two mutants, one in EMC5 and three in EMC2, are colored in blue, and show destabilizing phenotypes for EMC integrity.

D) A cytoplasmic cap structure involving EMC4 is conserved in yEMC and hEMC. Shown is a side-by-side comparison between the cytoplasmic domains of hEMC (left) and yEMC (right), highlighting the similar path EMC4 takes from the cytoplasmic domain towards the transmembrane domain. While an interaction surface between EMC8/9 and the EMC4 N-terminus is absent in yeast, yEMC4 binds at the top of the EMC2 TPR domain and assumes as similar position across the EMC3 cytoplasmic domain at the cytoplasm-membrane interface.

E) Location of two EMC2-EMC4 interfaces targeted for mutagenesis. hEMC is shown as surface rendering with the mutated EMC2 regions highlighted in blue.

F) Fluorescent client reporter stability assay for TMEM97 (N-cytoplasmic polytopic client), B1AR (N-lumenal polytopic client) and SQS ${ }^{378-410}$ (Clumenal tail-anchored client) in EMC2 KO cells expressing mutant hEMC2 ${ }^{\mathrm{E} 146 \mathrm{~A}+\mathrm{E} 149 \mathrm{~A}+\mathrm{Q} 150 \mathrm{~A}}$ (shaded) or WT hEMC2 rescue (unshaded).

G) Fluorescent client reporter stability assay, as in $\mathrm{F}$, for the hEMC2 $2^{\mathrm{E} 168 \mathrm{~A}+\mathrm{D} 170 \mathrm{~A}+\mathrm{K} 173 \mathrm{~A}}$ mutant. 


\section{Two distinct cavities are present in the transmembrane domain}

The transmembrane core of EMC is predicted to include contributions from each subunit except for EMC2 and, in humans, EMC8/9 (Figure 2C-D). The EMC presents two distinct and structurally conserved cavities on opposite sides of the transmembrane core that differ in size, shape, subunit compositions and apparent function (Figure 4A-B). One cavity, which we refer to as the lipid-filled cavity, appears contiguous with the ER lipid environment (Figure 4A). The second cavity, which we refer to as the gated cavity, appears to open towards the cytoplasm in our structures and is more occluded by a transmembrane helix gate from the lipid environment (Figure 4B). Notable structural hallmarks present in both species include a superimposable core of nine transmembrane helices, a set of flexible gate helices, and an amphipathic EMC1 brace helix (Figure 4C). 


\section{FIGURE 4}

A lipid-filled cavity

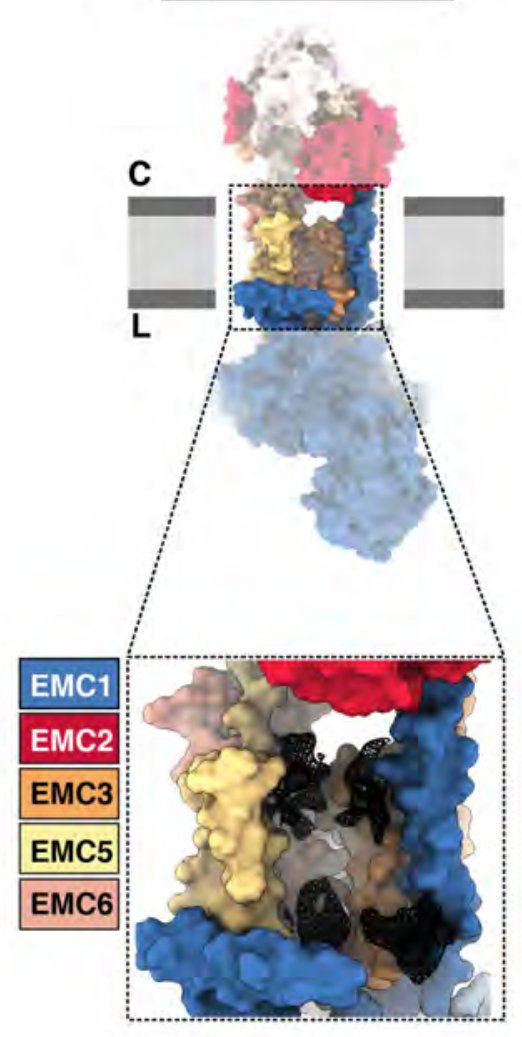

B

gated cavity

C

\section{hEMC (nanodiscs) yEMC (nanodiscs)}
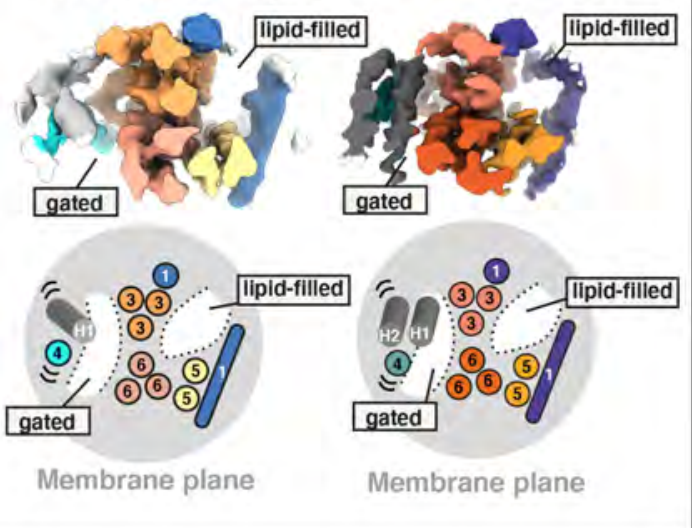

Membrane plane

Membrane plane

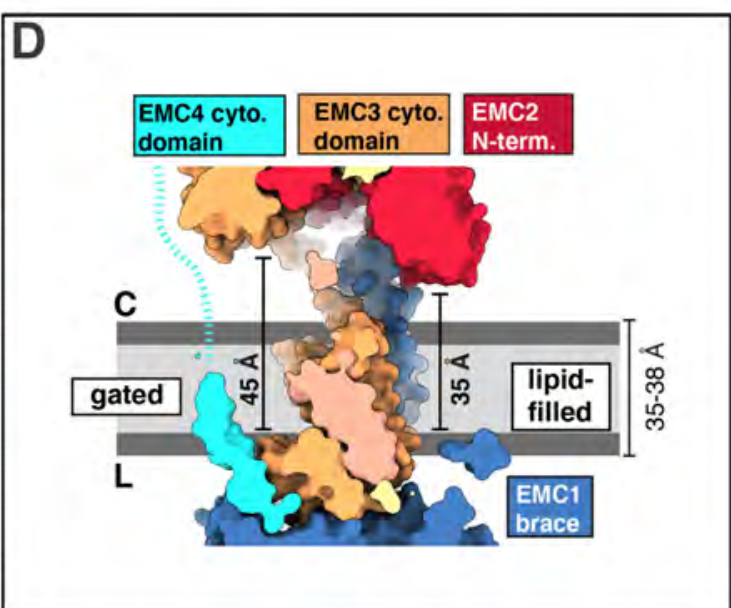

Figure 4: The EMC houses two transmembrane cavities with conserved core structures and distinct accessibilities.

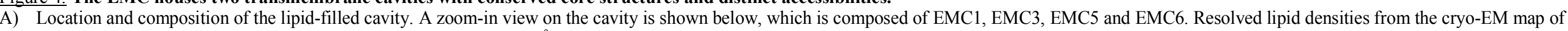
hEMC in POPC nanodiscs are shown as black mesh zoned within $3 \AA$ of modeled POPC molecules.

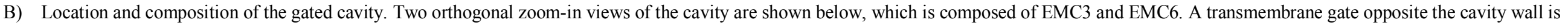

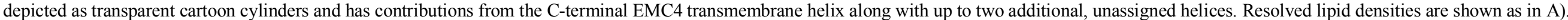

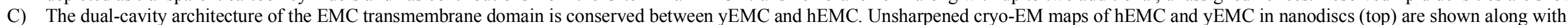

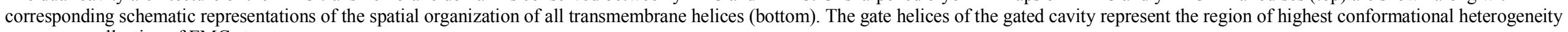
across our collection of EMC structures.

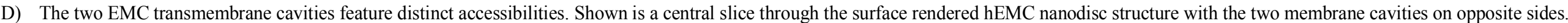

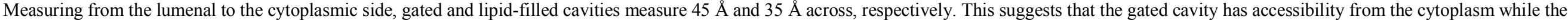
lipid-filled cavity does not. 


\section{The gated cavity serves as a conduit for terminal helix insertion}

Evaluating potential client paths from the cytoplasm into the transmembrane domain revealed a cavernous opening at the membrane-cytoplasmic interface of the gated cavity, wide enough to allow passage of a client helix, and tapering towards the lumen (Figure 4D). Consistent with its potential role as a cytoplasmic conduit into the EMC, the EMC3 portion of the cytoplasmic domain, which delineates this opening, sits approximately $45 \AA$ from the lumenal side of the gated cavity. This dimension exceeds the thickness of the ER membrane (Mitra, 2004; Heberle, 2020; Cornell, 2020) (Figure 4D). This cavity is lined primarily by EMC3, EMC4 and EMC6 (Figure 5A). Simulating the dimension of the first transmembrane helix of a known terminal insertase-client (B1AR, Chitwood, 2018) suggests that there is sufficient space for a client helix even in the client-free state of the EMC (Figure 5B). The gated cavity is hydrophilic on the cytoplasmic side and becomes increasingly hydrophobic towards the lumenal side (Figure 5C).

The entrance into the gated cavity interior (Figure 5A) is formed primarily by the EMC3 cytoplasmic domain. To test its function, charge swap mutations were introduced along the rim of this opening (hEMC3 $3^{\mathrm{E} 63 \mathrm{~K}}+\mathrm{D} 213 \mathrm{~K}+\mathrm{E} 223 \mathrm{~K}, \mathrm{hEMC} 3^{\mathrm{R} 59 \mathrm{E}+\mathrm{R} 62 \mathrm{E}+\mathrm{K} 216 \mathrm{E}}$ ) (Figure 5D, Figure 5 - figure supplement 2). These mutants resulted in loss of the tail-anchored client (SQS ${ }^{378-410}$ ) and partial loss of the N-terminal insertase dependent polytopic client (B1AR), reflecting a failure to support insertase activity. These mutants had no appreciable effect on the abundance of the polytopic transmembrane client (TMEM97) reporter (Figure 5E, Figure 5 - figure supplement 1-2). A similar phenotype was observed with alanine substitutions for a pair of lysines at the periphery of this cytoplasmic rim $\left(\mathrm{hEMC} 3^{\mathrm{K} 42 \mathrm{~A}+\mathrm{K} 43 \mathrm{~A}}\right)($ Figure 5 - figure supplement 2).

Having identified a functionally important entry route for terminal helix insertase clients, we next considered potential surfaces inside the cavity that might accommodate a client helix. A polar patch close to the membrane interior of this cavity was conspicuous, even though the specific amino acid residues are not strictly conserved (Figure 5 - figure supplement 2, Figure 1 - figure supplement 7). Mutating a pair of adjacent asparagine residues to equivalently sized but negatively charged aspartates $\left(\mathrm{hEMC}^{\mathrm{N} 114 \mathrm{D}+\mathrm{N} 117 \mathrm{D}}\right)$ resulted in a dramatic decrease in $\mathrm{SQS}^{378-410}$ and a mild decrease in the polytopic terminal insertase client (B1AR) (Figure 5F). Meanwhile, mutating a neighboring positively charged residue to an alanine (hEMC3 ${ }^{\mathrm{R} 180 \mathrm{~A}}$ ), a residue that is conserved in some of the YidC-superfamily insertase proteins (Figure 5 - figure supplement 4) (Anghel, 2017), resulted in partial loss of only the tail-anchored insertase client (SQS ${ }^{378-410}$ ) (Figure 5 - figure supplement 1). 
Lastly, we surveyed residues closer to the hydrophobic lumenal side of the gated cavity. Lipid density was resolved at positions along the cavity in hEMC and yEMC cryo-EM maps (Figure 4B) and the properties of this hydrophobic seal to the lumen are conserved (Figure 5 - figure supplement 2). The importance of this hydrophobic seal is suggested by the strong effect of a structurally-mild mutation of a conserved methionine to a leucine (hEMC $3^{\mathrm{M} 151 \mathrm{~L}}$ ), which caused significant decrease in both $\mathrm{SQS}^{378-410}$ and B1AR abundance (Figure 5G). Mutation of a neighboring aromatic residue $\left(\mathrm{hEMC} 3^{\mathrm{F} 148 \mathrm{~L}}\right)$, contacting both a lipid and the EMC4 C-terminal transmembrane helix, caused a marked decrease in all three client types without altering the levels of EMC subunits (Figure 5 - figure supplement 1-2, Figure 1 figure supplement 7). Together these results indicate that proper EMC insertase function depends on the exact composition of the cavity and not simply on its hydrophobic nature. 
FIGURE 5

A

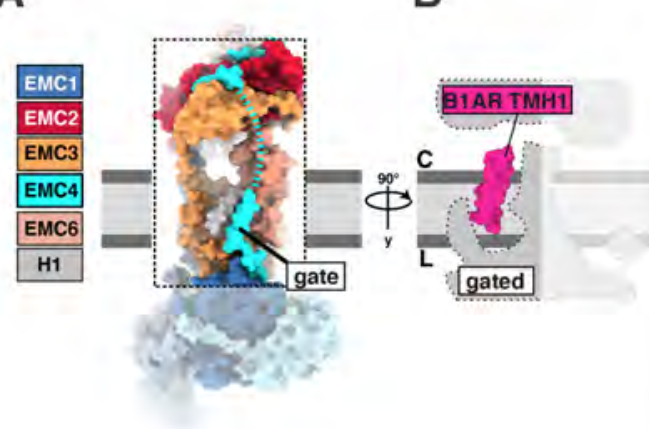

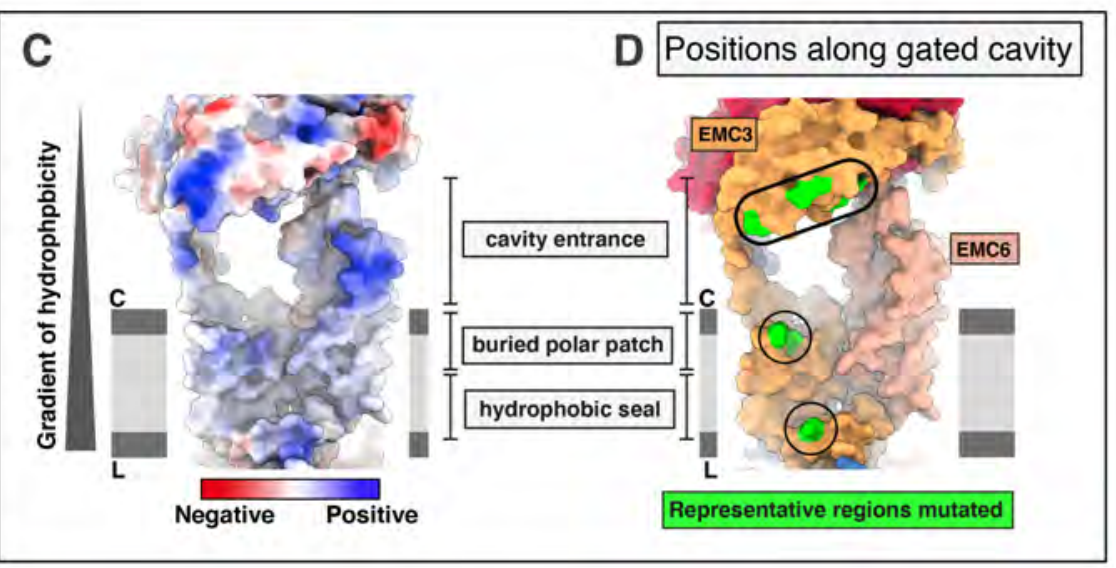
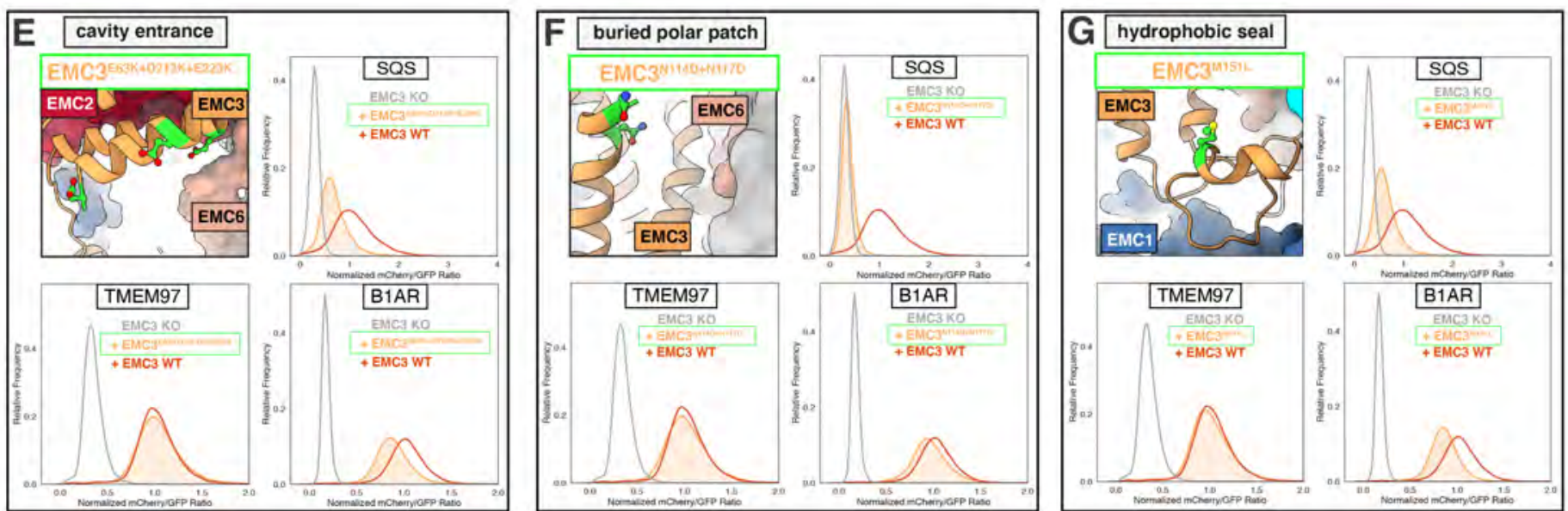

Figure 5: EMC houses an insertase module centered on EMC3 in the gated membrane cavity.

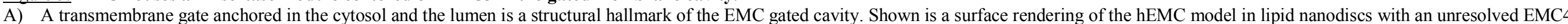
connection between the cytoplasm and the membrane depicted as a dashed line. An unassigned helix of the gate is shown in gray (H1).

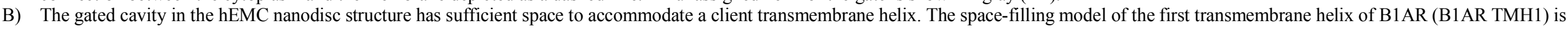
shown placed inside an outline of the gated EMC cavity.

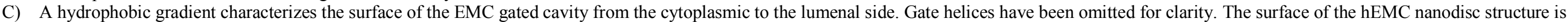
colored by electrostatic surface potential ranging from -15 (red) to +15 (blue) $\mathrm{kcal} /(\mathrm{mol} \cdot e)$.

D) Distinct EMC3 regions along the gated cavity hydrophobic gradient targeted for mutagenesis. Mutated residues are colored in lime.

E) Fluorescent client reporter stability assay for the EMC3 cavity entrance mutant, hEMC3 ${ }^{\mathrm{E} 63 \mathrm{~K}+\mathrm{D} 213 \mathrm{~K}+\mathrm{E} 223 \mathrm{~K}}$. A zoom-in on the mutated residues (colored lime) is shown in the top-right panel.

F) As in E) for the EMC3 buried polar patch mutant, hEMC3 $3^{\mathrm{N} 114 \mathrm{D}+\mathrm{N} 117 \mathrm{D}}$

G) As in E) for the EMC3 hydrophobic seal mutant, hEMC3 ${ }^{\mathrm{M} 151 \mathrm{~L}}$. 


\section{Structural heterogeneity suggests a role for the gate in regulating access to the insertase transmembrane cavity}

While the core transmembrane helices of the gated cavity are superimposable in all four of our EMC structures, the adjacent gate helices appear in different relative orientations. The structural variability likely reflects dynamics of the gate (Figure 4C). Comparing detergent and nanodisc maps for both species identified two major gate conformations (Video 2). One of the conformations, referred to as the closed gate conformation, results in a more occluded membrane cavity. The other conformation, referred to as the open gate conformation, would provide space for client accommodation.

The C-terminal transmembrane helix of EMC4 and ensuing lumenal segment are well resolved in all four structures; however, other regions of EMC4, including the segment connecting the cytoplasmic domain to the transmembrane gate helices, were poorly resolved perhaps owing to mobility. The yEMC detergent map, yEMC nanodisc map, and hEMC detergent map all show the unassigned helices in the closed conformation, preventing client residence in the gated cavity. By contrast, the hEMC nanodisc map reveals an open gate conformation with the unassigned helices shifted away from the transmembrane core to provide space for a client (Figure 5B). We note that the conformational heterogeneity and concomitant lower resolution of the gate likely accounts for the challenges in making unambiguous subunit assignments (Figure 5 - figure supplement 3), reflected by the three different interpretations reported in recent structures (Pleiner, Tomaleri, and Januszyk, 2020; O’Donnell, Phillips, and Yagita, 2020; Bai, 2020).

Considering the apparent flexibility of the gate, we sought to mutate the EMC4 interfaces resolved in the cytoplasm versus the membrane. As described above, mutating residues that together form a composite binding surface for the cytoplasmic domain of EMC4 (hEMC2 ${ }^{\mathrm{E} 146 \mathrm{~A}+\mathrm{E} 149 \mathrm{~A}+\mathrm{Q} 150 \mathrm{~A}}, \mathrm{hEMC2} 2^{\mathrm{E} 168 \mathrm{~A}+\mathrm{D} 170 \mathrm{~A}+\mathrm{K} 173 \mathrm{~A}}$, Figure 3F), we observed a modest accumulation of the tail-anchored insertase client $\left(\mathrm{SQS}^{378-410}\right.$ ) (Figure 3G, Figure 3 - figure supplement 1-2). Likewise, mutating residues in the center of the gated cavity, close to one of the unassigned helices in the closed gate conformation (hEMC3 ${ }^{\mathrm{V} 118 \mathrm{~A}+\mathrm{I122} \mathrm{A}}$ ) (Video 2, Figure 5 - figure supplement 1) led to an increase of $\mathrm{SQS}^{378-410}$. This SQS ${ }^{378-410}$ accumulation effect stands in contrast to mutating a residue that contacts the lumenal anchor of EMC4 (hEMC3 ${ }^{\mathrm{F} 148 \mathrm{~L}}$ ), which caused a reduction of $\mathrm{SQS}^{378-410}$ levels (Figure 5 - figure supplement 1). 


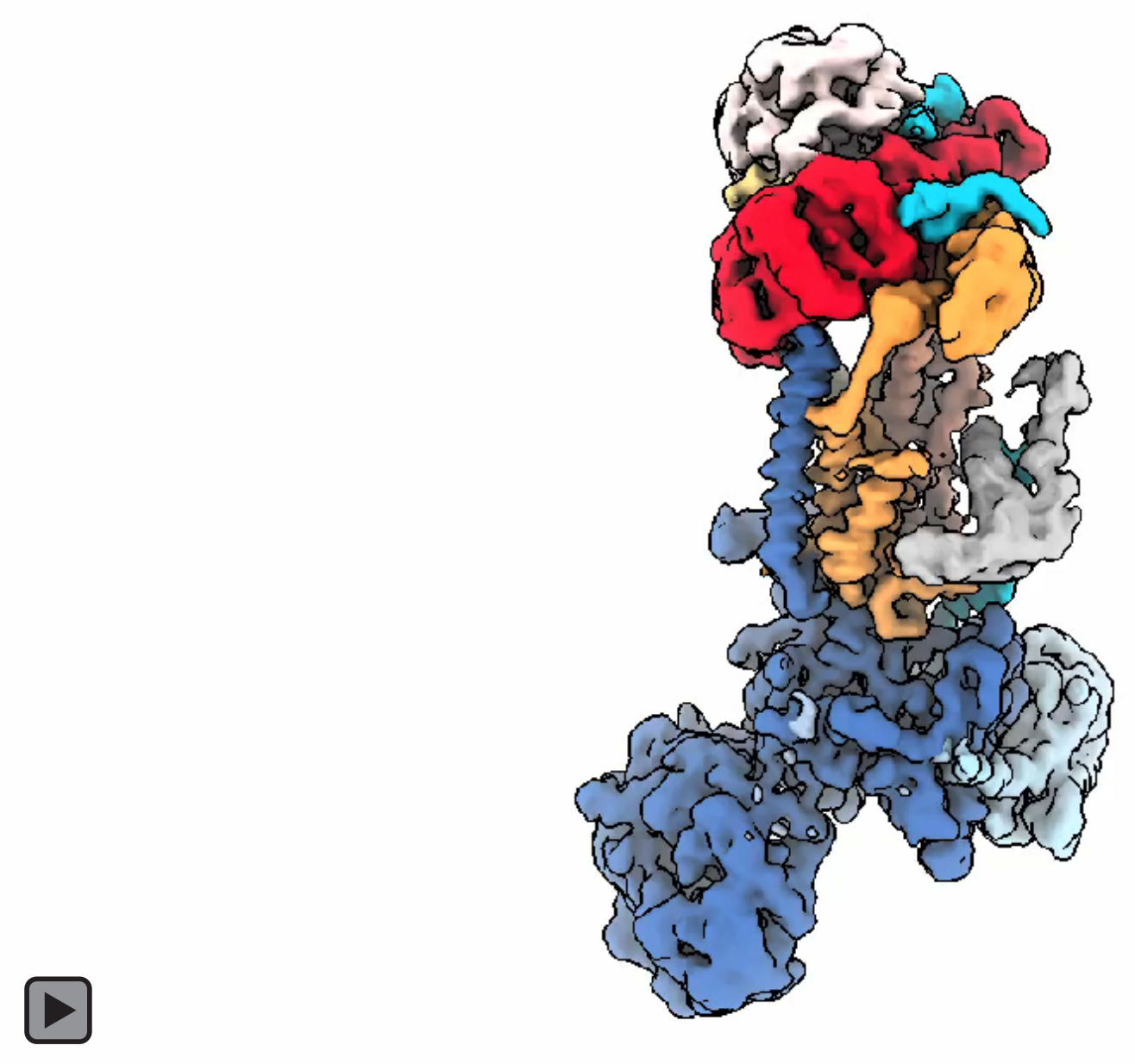

Video 2: EMC transmembrane cavity gate conformations

Overview of hEMC colored and labeled by subunit. Volume fades away to hEMC nanodisc model. hEMC nanodisc model remains constant as segmented maps of the unassigned gate helices are shown of hEMC detergent, yEMC detergent, and yEMC nanodisc maps. hEMC is colored cyan, yEMC is colored dark cyan, and gate helices are colored in shades of grey and purple as indicated by the label on the left. Two residues (hEMC3 N114 and N117) are shown in stick representation colored magenta and one residue (hEMC3 R180) is colored in gold. 


\section{The lipid-filled cavity is critical for both insertase-dependent and insertase-independent EMC functions}

In addition to the gated cavity, the EMC harbors another membrane-accessible cavity. The surface of the lipid-filled cavity includes contributions from EMC1, EMC3, EMC5 and EMC6 (Figure 6A). In our structures the EMC2 N-terminus occludes cytoplasmic accessibility to this cavity (Figure 4D, Figure 6A-B). However, this cavity may be accessible from the membrane or the ER lumen. The respective distance from the cytoplasmic EMC2 Nterminus to the lumenal side of the lipid-filled cavity is approximately $35 \AA$ across, which is close to the average ER membrane thickness (Mitra, 2004).

The lipid-filled cavity features a uniformly hydrophobic surface (Figure 6C) and superimposes across our ensemble of EMC structures. As noted, we resolved several lipids in our cryo-EM maps lining the cavity wall and modeled four POPC (1-palmitoyl-2-oleoyl-snglycero-3 phosphatidylcholine) molecules in the hEMC nanodisc map (Figure 6C). The residues in close proximity to these lipids are moderately conserved (Figure 6 - figure supplement 1). To characterize the functional role of the lipid-filled cavity, we mutated cavity-lining and lipid-proximal residues (Figure 6D, Figure 6 - figure supplement 2-3). Most of these mutations resulted in an increased abundance of the tail-anchored reporter $\left(\mathrm{SQS}^{378-410}\right)$ and mild decrease in production of polytopic client reporters (B1AR, TMEM97). However, one lipid-proximal mutant showed decreased levels of all three client reporter types with varying severity (hEMC3 ${ }^{\mathrm{R} 13 \mathrm{E}}$ ) without altering overall EMC levels. An analogous mutation in drosophila EMC3 was recently was reported to cause reduced levels of Rh1 in this mutant background (Xiong, 2020). The amphipathic EMC1 brace helix, which packs against the transmembrane helices of EMC5, is a structural hallmark of the lipid-filled cavity, (Figure 6D). Here, mutating interfacial residues from EMC5 (EMC5 $\left.{ }^{\mathrm{H} 19 \mathrm{~L}+\mathrm{S} 23 \mathrm{~A}+\mathrm{Q} 26 \mathrm{~L}}\right)$ caused a marked decrease in the N-lumenal polytopic reporter (B1AR) and a mild decrease in the tailanchored client (SQS ${ }^{378-410}$ ) with no effect on the TMEM97 reporter (Figure 6F).

Unexpectedly, mutating interfacial residues from EMC1 $\left(\mathrm{EMC1}{ }^{\mathrm{F} 437 \mathrm{Y}+\mathrm{R} 487 \mathrm{~K}}\right)$ showed a diametrically opposed phenotype in which B1AR was unaffected, SQS ${ }^{378-410}$ accumulated and TMEM97 levels markedly decreased (Figure 6G). Two other interfacial brace mutants which had yet different resulting client flow cytometry profiles (Figure 6 - figure supplement 3 BC). The pleiotropic client phenotypes across the panel of interfacial brace mutants suggest that this feature is critical for multiple EMC functions. 


\section{FIGURE 6}
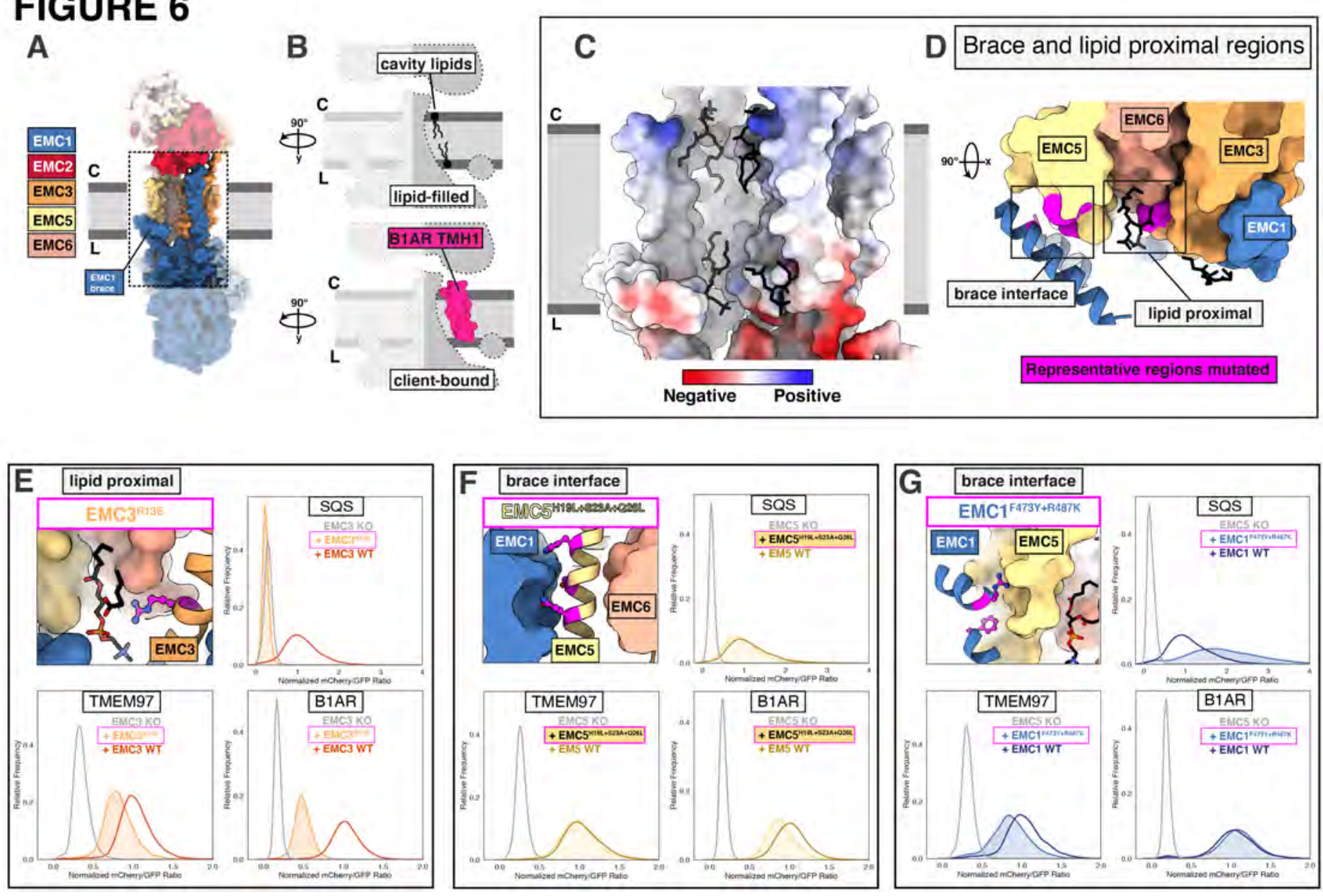

Figure 6: A lipid-filled cavity in the EMC transmembrane domain stabilizes disparate client proteins.

A) An EMC1 amphipathic brace helix delineates the boundary of the lipid-filled transmembrane cavity and packs against EMC5. Shown is a surface rendering of the hEMC model in nanodiscs. EMC4, EMC5, EMC6, and EMC1 subunits all contribute to the cavity lining.

B) The lipid-filled cavity in the hEMC nanodisc is occupied by several lipid molecules. Cartoon outlines of the gated cavity illustrate that the cavity could in principle allow for occupancy of a client helix (B1AR TMH1), possibly by lipid displacement or movement of the EMC1 brace helix.

C) The lipid-filled cavity has a uniform hydrophobic lining. Shown is an electrostatic surface rendering of the hEMC nanodisc structure colored as in Figure 5C. The cytoplasm-membrane interface contains positively charged residues and the lumenal interface contains negatively charged residues. Modeled phospholipid molecules are displayed in black.

D) Lipid-proximal and brace interface residues targeted for mutagenesis. Selected regions targeted for mutagenesis are colored in magenta and include brace interface mutations both in EMC1 and EMC5, as well as a lipid-proximal residue in EMC3.

E) Fluorescent client reporter stability assay for the $\mathrm{hEMC} 3^{\mathrm{R} 13 \mathrm{E}}$ mutant, which is in close proximity to a modeled POPC molecule.

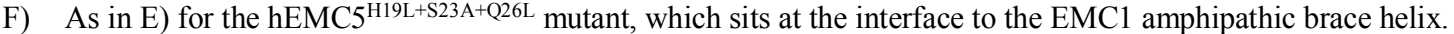

G) As in E) for the $\mathrm{hEMC1}{ }^{\mathrm{F} 473 \mathrm{Y}+\mathrm{R} 487 \mathrm{~K}}$ mutant, which sits at the interface to the EMC5 transmembrane helices. 


\section{The EMC lumenal domain is crucial for the biogenesis of multi-pass transmembrane proteins}

Composed primarily of EMC1, EMC7 and EMC10, the extensive EMC lumenal domain (Figure 7A) is important for polytopic client biogenesis and interactions with lumenal chaperones (Luo, 2002; Shurtleff and Ithzak, 2018; Hiramatsu, 2019; Coehlo, 2019). EMC7 and EMC10 are scaffolded on two beta-propellers of EMC1, one distal and the other proximal to the membrane. The lumenal cap differs between hEMC and yEMC, with a four-bladed distal beta-propeller in yeast and eight-bladed distal propellor the human complex (Figure 7B). All three lumenal EMC subunits have structural folds known to participate in proteinprotein interactions (Reinisch \& De Camilli, 2017). Mutations in this lumenal domain have been linked to loss of the EMC complex (Bircham, 2011), a trafficking delay for membrane protein Pmal (Luo, 2002), and male infertility (Zhou, 2018).

Several regions of the lumenal domain form stabilizing interactions with the membrane cavities. The gate helices of the gated cavity are anchored via the embedding of EMC4's Cterminus within the membrane-proximal EMC1 propeller. The lipid-filled cavity is connected to the ER lumenal domain via the amphipathic EMC1 brace helix, which is tethered to the membrane-proximal EMC1 beta-propeller. The connections between the lumenal domain and the transmembrane cavities could allow for conformational coupling during client handling. Indeed, superimposing the two conformations presented above, the open and closed gate states, revealed not only differences in the transmembrane domain but also a rotation of the lumenal domain relative to the membrane cavities (Video 3). The lumenal positioning is consistent for all three of our closed gate conformation reconstructions (hEMC detergent, yEMC nanodisc, yEMC detergent). By contrast the one map with an open gated cavity displayed a lumenal rotation and concomitant shifts in position of the EMC1 brace helix (Figure 7 - figure supplement 1). Indeed, our set of interfacial EMC1 brace mutants described above (Figure 6F-G, Figure 6 - figure supplement 3), showed differing client phenotypes when mutated from either the EMC1 or the EMC5 side. This suggests a complex conformational interplay between lumenal and transmembrane domains during the engagement of diverse client types.

We investigated several known disease mutations in both conserved and human-specific regions of EMC1 (Figure 7C-E, Figure 7 - figure supplement 2-3) (Harel, 2016; AbuSafieh, 2013; Amberger, 2019). One of these disease-associated residues sits near the anchor point for the lumenal EMC4 transmembrane gate helix $\left(\mathrm{hEMC1} 1^{\mathrm{R} 81 \mathrm{C}}\right)$, while the majority are found farther from the membrane (hEMC1 ${ }^{\mathrm{G} 868 \mathrm{R}}, \mathrm{hEMC1}^{\mathrm{A} 144 \mathrm{~T}}, \mathrm{hEMC} 1^{\mathrm{T} 82 \mathrm{M}}$ ) (Figure 7C-E). Incorporating each of these disease mutations into our EMC functional assay resulted in lower levels of the N-cytoplasmic polytopic client (TMEM97) and an increase in the level of the tail-anchored client $\left(\mathrm{SQS}^{378-410}\right)$, discussed in more detail below.

Two different EMC1 mutants associated with cerebellar atrophy, visual impairment, and psychomotor retardation ( $\mathrm{hEMC1} 1^{\mathrm{T} 82 \mathrm{M}}, \mathrm{hEMC1}^{\mathrm{G} 868 \mathrm{R}}$ ), map to the hinge region between the EMC1 beta propellers where EMC7 binds (Figure 7D). Both of these mutants, at this proteinprotein interface, result in depletion of the N-cytoplasmic polytopic client (TMEM97). EMC7 
and EMC10 form beta-sandwich domains on either side of the membrane-proximal betapropeller of EMC1, and contact each other across the EMC1 surface. Consistent with our structures, coupling of these subunits is supported by the prior finding that in the absence of EMC7, EMC10 is also lost from the complex while the other EMC components appear unaffected (Shurtleff and Ithzak, 2018). EMC7 and EMC10 have been proposed to be auxiliary components with weaker phenotypes compared to core EMC subunits (Jonikas, 2009; Shurtleff and Ithzak, 2018; Dickinson, 2016). Upon deleting EMC7, multi-pass transmembrane clients are retained in the ER but tail-anchored clients decrease in abundance (Shurtleff and Ithzak, 2018).

Several features of our data suggest dynamic association of EMC7. Density for the EMC7 beta-sandwich at the hinge between the two EMC1 beta propellers was relatively weak in the consensus hEMC nanodisc map (Figure 2 - figure supplement 4). Additional rounds of 3D classification revealed two distinct classes, one with clear density for EMC7 and one with weak density in this region. Mass spectrometric analysis of purified hEMC, however, revealed that the abundance of EMC7 was similar to that of the other EMC components (Figure 1 - figure supplement 2; Source Data 1 - Supplementary Table 3). Both reconstructions, with and without density for the EMC7 lumenal domain, displayed wellresolved density for EMC10. Together, we conclude that EMC7 is associated with EMC1 in two different conformational states of EMC7 with potentially distinct functions.

The OMIM database (Amberger, 2019) lists a mutation of unknown significance linked to retinitis pigmentosa $\left(\mathrm{hEMC1}^{\mathrm{A} 144 \mathrm{~T}}\right)$ residing in the EMC1 distal propeller (Figure 7E).

Additionally, we also generated mutations in two surface exposed patches of the membranedistal EMC1 beta-propeller projecting into the lumen (hEMC1 ${ }^{\mathrm{R} 69 \mathrm{D}}, \mathrm{hEMC1}{ }^{\mathrm{G} 71 \mathrm{~S}}, \mathrm{hEMC1}^{\mathrm{H} 93 \mathrm{D}+}$ E138D + N282K, Figure 7 - figure supplement 2-3). Overall, these mutations displayed the same client effect: a decrease in the N-cytoplasmic polytopic client reporter (TMEM97), no change in the N-lumenal polytopic client reporter (B1AR), and accumulation of the tail-anchored client reporter $\left(\mathrm{SQS}^{378-410}\right.$ ) (Figure 7 - figure supplement 2-3). Upon identifying antibodies against yEMC we observed that the top two antibodies bind to a similar extended loop in the distal yEMC1 beta-propeller, perhaps suggesting that this site is accessible for co-factor binding in the ER. Intriguingly, this region of the lumenal domain corresponds to the region where hEMC1 has an expanded distal beta-propeller. Taken together, the data provide evidence that the lumenal domain is functionally coupled to the broader EMC role in transmembrane client stabilization. Moreover, these data support that the EMC is acting as a holdase chaperone to shield polytopic clients from degradation while they are folding to their functional form. 


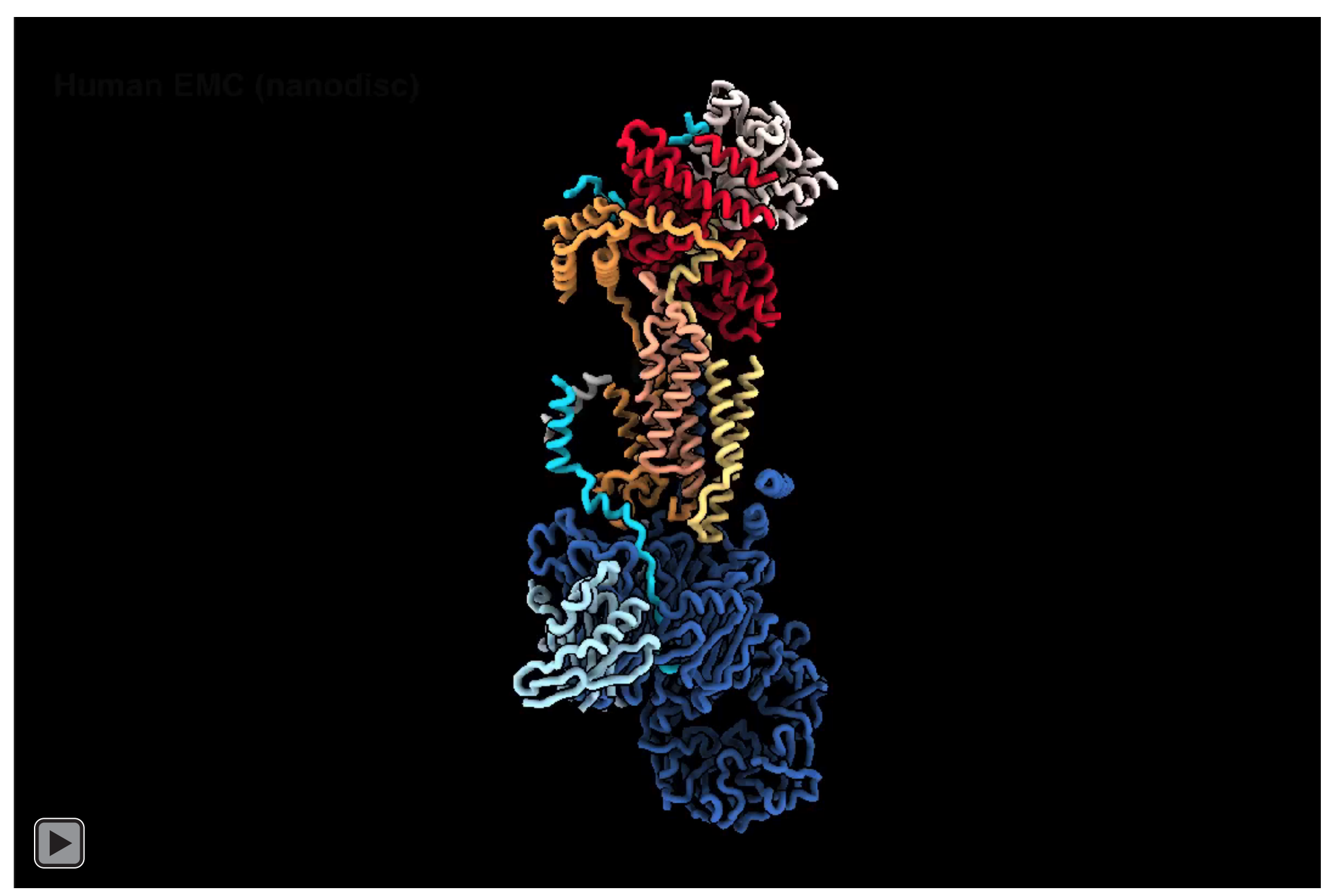

Video 3: hEMC lumenal domain differences between nanodisc and detergent models

Overview of hEMC nanodisc model colored and labeled by subunit. Structural landmarks are labeled. hEMC detergent model (colored grey) fades in and both models rotate. As the models rotate several structural features are highlighted. 
bioRxiv preprint doi: https://doi.org/10.1101/2020.09.02.280008; this version posted September 19, 2020. The copyright holder for this preprint (which was not certified by peer review) is the author/funder, who has granted bioRxiv a license to display the preprint in perpetuity. It is made available under aCC-BY-NC-ND 4.0 International license.

FIGURE 7

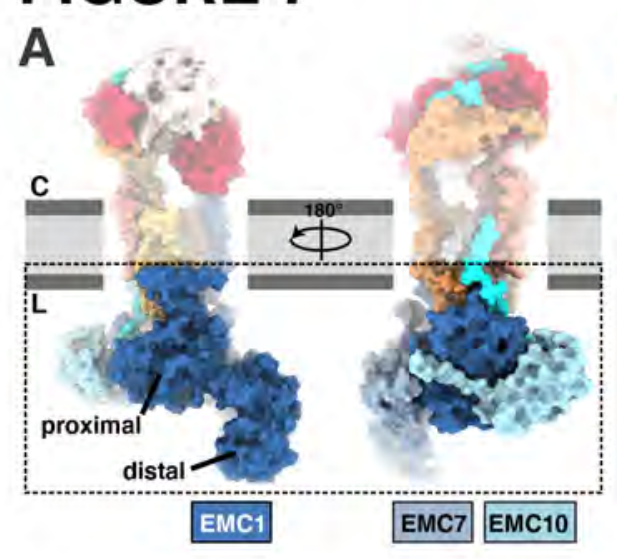

B Distal propellor comparison between hEMC and yEMC1

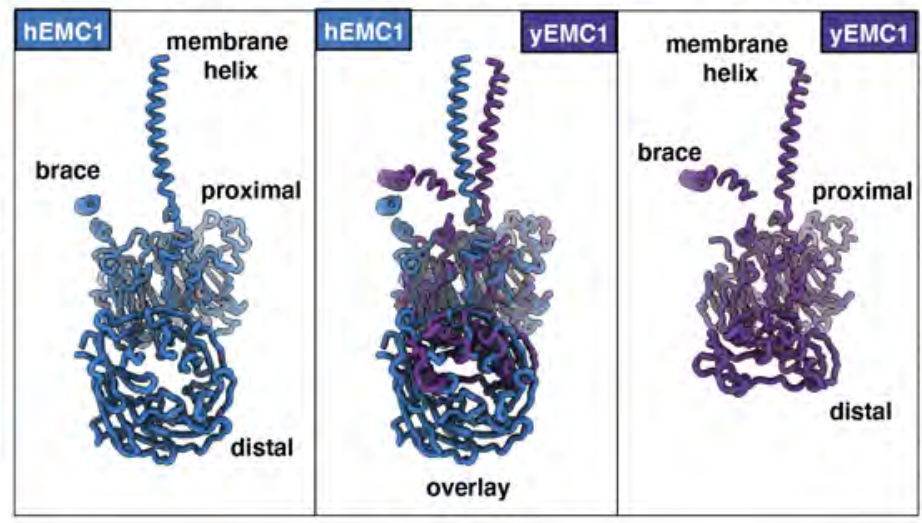

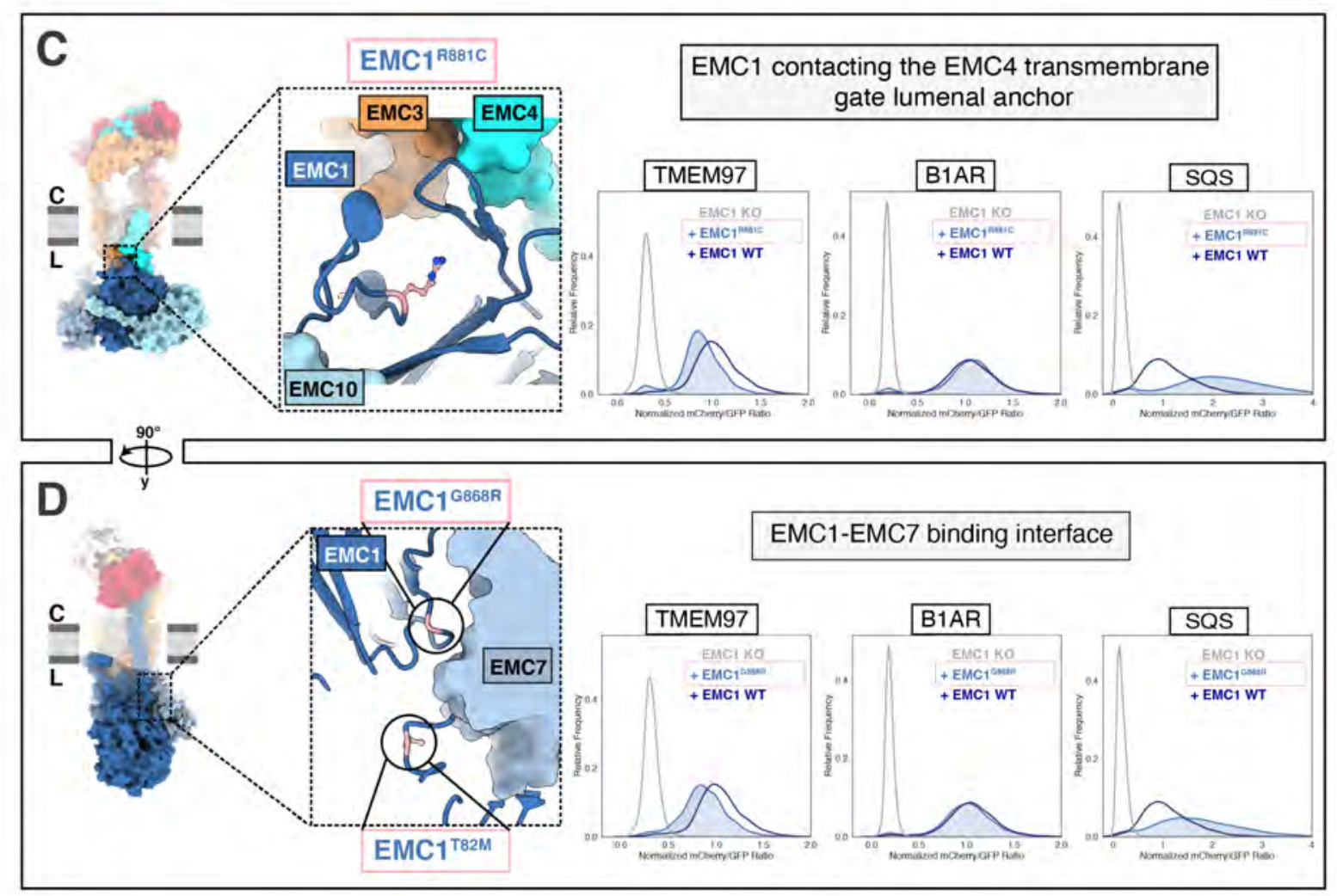

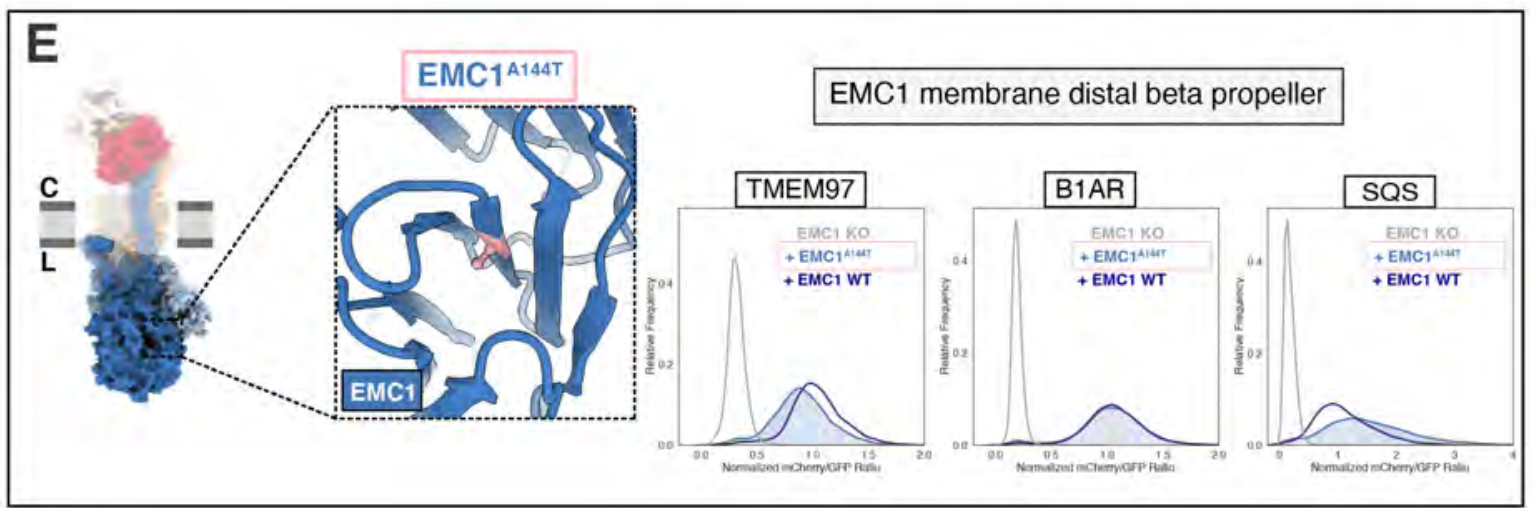

Figure 7: The large EMC lumenal domain is the site for several annotated disease mutations.

A) Two views of the hEMC nanodisc structure. Two beta propellers are present in EMC1, one proximal to the membrane and one distal.

B) EMC1 is the largest EMC subunit and differs in size between yeast and human. Shown are human EMC1 (nanodisc), an overlay of human and yeast EMC1 (both nanodisc), and yeast EMC1 (nanodisc).

C) The hEMC1 ${ }^{\mathrm{R} 881 \mathrm{C}}$ mutant sits near the EMC4 lumenal gate anchor. Left: Location of the mutation (colored pink). Right: Fluorescent client reporter stability assay for $\mathrm{hEMC1} \mathrm{R}^{\mathrm{R} 881 \mathrm{C}}$.

D) As in C) for the hEMC ${ }^{\mathrm{G} 868 \mathrm{R}}$ mutant.

E) As in C) for the hEMC ${ }^{\mathrm{Al} 44 \mathrm{~T}}$ mutant. 


\section{$\underline{\text { Discussion }}$}

Our collection of yeast and human EMC structures revealed the intricate and dynamic architecture of this multifunctional transmembrane molecular machine. The structures served as the starting point for our systematic dissection of EMC's multifaceted functions by exploring the impact of structure-based mutations on the ability of the EMC to support the biogenesis of representative members of three classes of membrane proteins: SQS, a tail-anchored protein, which exploits EMC's C-terminal insertase activity; B1AR, which relies on EMC's N-terminal insertase activity; and TMEM97, a polytopic membrane protein, which depends on the EMC for its biogenesis but does not rely on either of EMC's terminal insertase activities. Our data revealed that a conserved dual membrane cavity architecture supports the biogenesis of this diverse panel of transmembrane clients.

Overall, our studies present a nuanced picture of EMC's multifunctionality, revealing structural regions that differentially impact production of the three distinct client types. Unexpectedly, we also find that alterations to either the cytoplasmic or lumenal domain of EMC lead to enhanced abundance of the TA substrate. Moreover, our work provides a foundational framework for understanding how discrete yet allosterically coupled regions of the complex enable the multiple functions of the EMC to support membrane protein biogenesis. Taken together these studies suggest a model in which the EMC differentially regulates the biogenesis of distinct membrane proteins, thereby contributing to cellular coordination of membrane protein abundance in accordance with physiological needs. We propose a model of the EMC functioning both as a terminal insertase as well as a holdase chaperone that is potentially modulated by post-translational modifications, lipid interactions, and protein-protein interactions [FIGURE 8]. 
bioRxiv preprint doi: https://doi.org/10.1101/2020.09.02.280008; this version posted September 19, 2020. The copyright holder for this preprint (which was not certified by peer review) is the author/funder, who has granted bioRxiv a license to display the preprint in
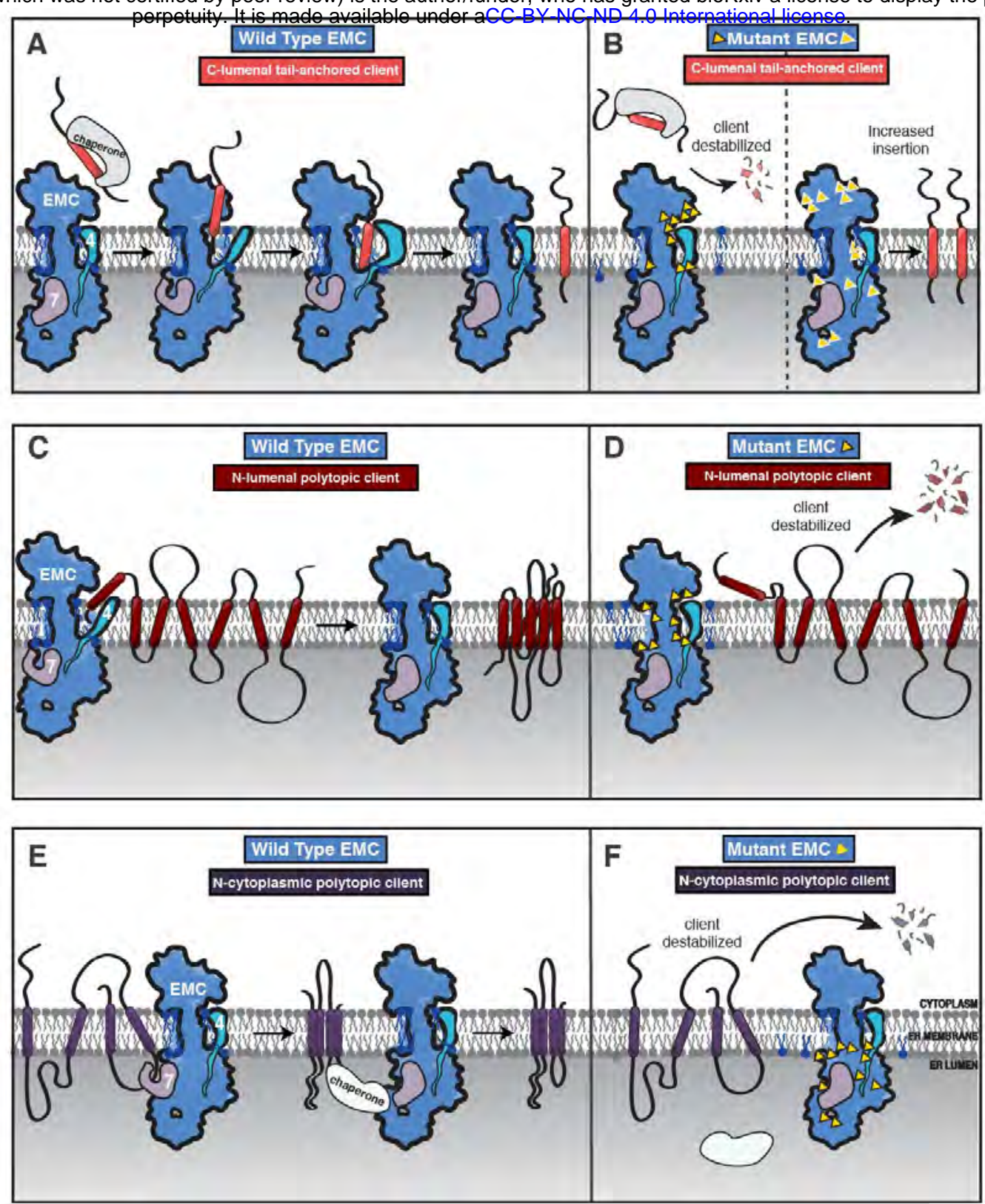

Figure 8: Model of coordinated EMC functions

A) Model of EMC insertase function for a C-lumenal tail anchored client. Cytosolic factors bring post-translationally localized clients to the ER. Then the client engages the EMC cytoplasmic domain. The polar roof modulates entry into the gated cavity. A hydrophobic slide facilitates the client helix fully entering the cavity. A lateral movement of the gate releases the client helix into the membrane and the EMC gate closes.

B) Our mutagenesis data provide the following insights into EMC regions of functional importance for each of the three client types we tested. Mutants are depicted by yellow triangles. Tail anchored client (coral) abundance was depleted upon mutagenesis of the cytoplasmic domain entrance to the gated cavity, polar and charged residues at the cytoplasm-membrane boundary, residues along the length of the gated cavity, in the hydrophobic seal to the lumen, and lipid interacting residues in both cavities (left). We also observed a subset of mutants that resulted in higher levels of the C-lumenal tail anchored client (right) that are positioned in the cytoplasmic domain cap, throughout the ER lumenal domain, and one mutation at the center of the gated cavity.

C) The EMC facilitates biogenesis of N-lumenal polytopic client protein B1AR (dark red).

D) Regions important for B1AR stability primarily map to the transmembrane region of the EMC structure, with depletion observed for lipid interacting residues on both sides of the cavity, the polar entrance roof of the gated cavity, and the EMC1 brace helix.

E) The EMC facilitates biogenesis of N-cytoplasmic polytopic client protein TMEM97 (dark purple).

F) Regions important for TMEM97 stability were primarily located in the lumenal domain spanning both propellers, in EMC1. In addition to these lumenal regions there was a depletion of TMEM97 at the lipid-interacting positions at the lumenal interface of both membrane cavities of the EMC. 


\section{Terminal insertase clients require an embedded insertase module within the EMC}

EMC3's fold at the interface between the cytoplasm and membrane forms the core of the gated cavity and is reminiscent of proteins from the YidC family of insertases (Borowska, 2015; Dalbey, 2015; Anghel, 2017). Indeed, mutations in either the cytoplasmic or transmembrane domains of EMC3 establish that these features are critical for terminal helix insertase activity. In light of our observation of multiple gate conformations, we speculate that these conformations modulate insertion and release into the ER membrane.

Notably, mutating the surface of the cytoplasmic cap, which extends beyond the EMC3 cytoplasmic helices towards EMC8/9, resulted in an unexpected increase in C-tail anchor client (SQS ${ }^{378-410}$ ) abundance. Of the three clients analyzed, SQS was the only one to show enhanced levels. It is unclear if this enhancement is SQS-specific or representative more broadly of all post-translationally targeted EMC tail-anchored clients. Future studies will be required to address if this is due to regulated insertion of SQS by the EMC, parallel pathways for inserting SQS into the membrane (i.e. mediated by TRC40/GET), and/or slower cytoplasmic clearance of chaperone-bound SQS.

\section{Both EMC cavities have resolved lipids and are critical for client biogenesis}

Both the N-terminal (B1AR) and C-terminal insertase (SQS) clients depend on the EMC gated cavity. Indeed, both the SQS tail-anchored helix and the first transmembrane helix of B1AR are moderately hydrophobic, with polar residues near the cytoplasmic end of the transmembrane helix, and both showed a strong dependence on the gated cavity. Nevertheless, our panel of mutants revealed some notable differences in the handling of these two client types. B1AR showed more dependence than SQS on the lipid-filled cavity in contrast to mutants elsewhere in the complex. One possible reason could be due to differences in the mechanism of initial engagement: SQS is targeted to the ER by cytoplasmic chaperones, while B1AR is targeted by SRP. Another key difference is that $\mathrm{B} 1 \mathrm{AR}$ is polytopic and needs to overcome the additional challenge of tertiary transmembrane packing to reach its folded state. Future work will address the interplay between B1AR synthesis and its co-translational engagement with the translocon versus the EMC.

\section{The EMC lumenal domain orchestrates holdase chaperone function important for}

\section{polytopic clients}


Unlike the two terminal insertase clients we investigated, TMEM97 biogenesis was negatively impacted by mutation of the lumenal EMC1. The depletion of TMEM97 observed in these mutant backgrounds is consistent with the lumenal domain contributing to a holdase chaperone function, passively shielding its client while it is being synthesized and/or folded (Zhang, 2017). Interestingly, the diametrically opposed phenotype of mutants in the EMC lumenal domain on SQS raises the possibility that occupancy by one type of client can support an EMC conformation that is unfavorable for receiving the other. Alternative conformations could establish competition between client types for EMC occupancy. One explanation for this observation is that there is a conformational change between the insertase-active versus the holdase-active states. Interestingly, we identified at least two EMC conformations in our collection of structures, and EMC may adopt different conformations in various client and cofactorengaged states.

In yeast, the polytopic clients co-purifying with the EMC are also glycosylated. One possible model is that the putative carbohydrate binding domains in EMC7 or EMC10 directly contribute to engagement with client proteins. We speculate post-translational modifications on clients and the EMC could modulate function including client binding, chaperone binding, or regulating signaling in response to cellular cues.

\section{Potential role of the EMC as a master regulator of membrane protein biogenesis as the}

\section{basis for its pleiotropic phenotypes}

Why does the cell use a multifunctional EMC molecular machinery rather than specialized machinery for each of the functions encompassed by the EMC? Considering that the cell already has general machinery (Sec61 translocon) and tail-anchor insertase machinery (GET/TRC complex), we speculate that the EMC coordinates biogenesis of diverse membrane proteins. Several observations suggest broader roles of the EMC as an integrator of information sensing the protein and lipid environment and coordinating its multiple activities, including the regulating the biogenesis of membrane proteins. For example, the initial identification of the EMC included numerous genetic interactions with both protein and lipid synthesis factors in yeast (Jonikas, 2009) and these disparate interdependencies have been subsequently observed in numerous species including human EMC (Lahiri, 2014; Tang, 2017; Guna, 2018; Volkmar, 2019; Volkmar, 2020). Also, several client proteins are enzymes or cofactors involved in multiple stages of lipid synthesis or trafficking, and this may provide a unifying explanation for the range of genetic interactions and co-essentiality observations reported to date (Guna, 2018; 
Shurtleff and Ithzak, 2018; Volkmar, 2018; Tian, 2019; Wainberg, 2019; Corradi, 2019; Volkmar, 2020). In this regard, one structural feature of particular interest is the EMC1 amphipathic brace, which resides adjacent to the lipid-filled cavity. This conserved feature sits within the interfacial membrane boundary, raising the possibility that it can modulate the lipid or protein composition of this cavity. Notably, several other membrane proteins involved in ER homeostasis, including Opi1 and Ire1, also contain amphipathic helices that have been proposed to sense the properties of the lipid bilayer (Volmer, 2013; Jacquemyn, 2017; Halbleib, 2017; Hofbauer, 2018; Cho, 2019). Future work will explore how the EMC overall, and the EMC1 brace helix in particular, govern client release into the membrane, interface with the local structure of the lipid bilayer, and play roles in specific client-lipid interactions.

In addition to the three client classes we investigate here, it is clear that EMC has a broader range of clients including multi-protein assemblies (Richard, 2013; Talbot, 2019), lipid modulating proteins (Volkmar, 2018), lipid binding proteins (Salas-Estrada, 2018; Sejdju, 2020), and those with helices that do not span the bilayer (Lin, 2019; Ngo, 2019). The compartmentalization and interdependence that we observe for effects of mutations on client handling provide a foundation for understanding this multifunctionality. We propose that the complexity of the EMC machine, combining insertase and holdase chaperone functions within one molecular machine, has arisen to mitigate the error prone biogenesis of a diverse range of membrane spanning proteins in the dynamic environment of the ER.

\section{Materials and Methods}

Reagents used for experiments described and reagents made as part of this study are listed in Supplementary Table 2.

\section{Cell Line Maintenance}

K562 dCas9 KRAB cells were grown in RPMI 1640 (GIBCO) with 25 mM HEPES, $2 \mathrm{mM}$ l-glutamine, $2 \mathrm{~g} / \mathrm{L} \mathrm{NaHCO}_{3}$ and supplemented with $10 \%(\mathrm{v} / \mathrm{v})$ fetal bovine serum (FBS), 100 units/mL penicillin, $100 \mu \mathrm{g} / \mathrm{mL}$ streptomycin, $2 \mathrm{mM}$ l-glutamine. HEK293T cells were grown in Dulbecco's modified eagle medium (DMEM, GIBCO) with $25 \mathrm{mM}$ d-glucose, $3.7 \mathrm{~g} / \mathrm{L} \mathrm{NaHCO}_{3}, 4 \mathrm{mM}$ l-glutamine and supplemented with $10 \%$ (v/v) FBS, 100 units $/ \mathrm{mL}$ penicillin, $100 \mu \mathrm{g} / \mathrm{mL}$ streptomycin. All cell lines were grown at $37^{\circ} \mathrm{C}$. All cell lines were periodically tested for Mycoplasma contamination using the MycoAlert Plus Mycoplasma detection kit (Lonza). 


\section{DNA transfections and virus production}

Lentivirus was generated by transfecting HEK39T cells with standard $4^{\text {th }}$ generation packaging vectors using TransIT-LT1 Transfection Reagent (Mirus Bio). Media was changed 10 hours post-transfection. Viral supernatant was harvested 60 hours after transfection, filtered through $0.45 \mu \mathrm{m}$ PVDF filters and frozen prior to transduction.

\section{Knockout EMC Cell Lines}

A single and dual knockout guide system was developed in the $\mathrm{pX} 458$ backbone (Addgene plasmid \# 48138) with guides targeting EMC1, EMC2, EMC3, or EMC5 (see reagents table). Targeting guides were selected using the Broad's guide selection tool (https://portals.broadinstitute.org/gpp/public/analysis-tools/sgrna-design). For the single EMC5 knockout system, an EMC5 targeting guide was cloned into pX458 by digesting with BbsI and ligating to annealed oligos for the EMC5 sgRNA. For the dual knockout system, a four-step cloning process generated the final knockout plasmid: 1) Each of the two guides targeting the same locus were individually cloned into $\mathrm{pX} 458$. 2) Then pX458_sgRNA1 was digested with XbaI 3) SgRNA2 cassette from pX458_sgRNA2 was PCR amplified with oligos containing overhangs spanning the XbaI cloning site and purified. 4) Finally, the final dual guide vector was generated by Gibson cloning (NEBuilder).

To generate the EMC knockout cell lines, K562 dCas9 KRAB cells were nucleofected with the respective EMC knockout plasmids using Lonza SF Cell Line 96-well Nucleofector ${ }^{\mathrm{TM}}$ Kit (V4SC-2096). Two days post nucleofection, GFP-positive cells were single cell sorted into 96-well plates using BD FACS ArialI. After colonies from single cells grew out, genomic DNA was isolated using QuickExtract (Lucigen), the sgRNAtargeted sites were PCR amplified and then NGS-sequenced via Genewiz's EZ-Amplicon service. Sequencing data was analyzed and aligned to the respective reference alleles in the human genome. Clones whose alleles harbored only indel mutations for EMC1, $\mathrm{EMC}$, EMC3, and EMC5 (full knockouts) respectively were further validated on the protein level.

\section{Dual fluorescent EMC client reporter cell lines}

Dual client reporters for TMEM97, ADRB1 (protein name: B1AR), and FDFT1 (protein name: SQS) were introduced lentivirally into each of the EMC1, EMC2, EMC3, and EMC5 knockout cell lines. TMEM97 and ADRB1 full length sequences were used with a C-terminal tag -mCherry-P2A-GFP. The sequence for FDFT1 transmembrane domain $\left(\mathrm{SQS}^{378-410}\right)$ was tagged N-terminally with GFP-P2A-mCherry- and an opsin tag on the C-terminus as used in a prior study (Guna, 2018). Three days post-transduction, $\mathrm{GFP} / \mathrm{mCherry}$ positive cells were sorted on BDAriaII. Sequences for these constructs are available in Supplementary Table 2. 


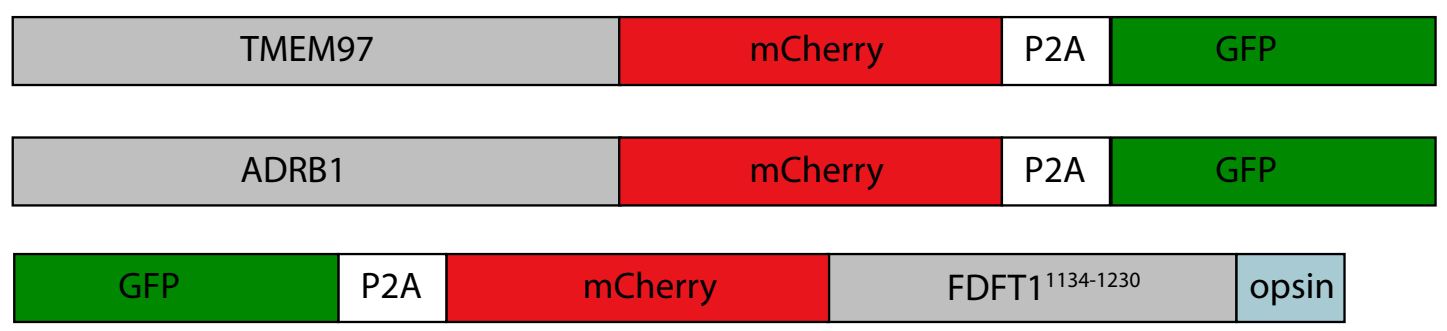

\section{Mutant EMC cell lines}

The EMC mutant genes were synthesized and cloned by Twist into pKDP119-SFFV[insert site]-IRES-Puro-P2A-BFP. For EMC subunit mutation details refer to the reagents table. Mutant EMC cell lines were generated by lentiviral introduction of the respective EMC mutant subunit into the respective knockout cell lines (EMC1, EMC2, EMC3 or EMC5) containing the dual fluorescent reporters for each EMC client (pKDP110_ADRB1_mCherry_P2A_GFP, pKDP111_TMEM97_mCherry_P2A_GFP, or GFP_P2A_mCherry_FDFT1_TMD_opsintag). The expression of each fluorescent reporter was read out 6 days after puromycin selection in each of the EMC mutant cell lines.

\section{EMC Subunit}

IRES

PuromycinR

$\mathrm{P} 2 \mathrm{~A}$

\section{Flow analysis}

For each EMC mutant cell line, 20000 live cells were recorded on Attune NxT flow cytometer. FlowCal flow analysis package was used for analysis in Python. First, live cells were gated based on FSC/SSC. Then GFP (BL1-A) and mChery (YL2-A) were plotted for each mutant and control cell line. mCherry:GFP intensity ratios were calculated for individual cells in each cell line. Fluorescence ratios for each substrate in an EMC mutant cell line were normalized to the mCherry:GFP ratio of the same substrate in the EMC wild type rescue cell line. Distributions of fluorescence ratios were plotted as histograms in Python using seaborn.

\section{Western Blotting}

Cell pellets were lysed using lysis buffer (20 mM Tris pH 7.5, $150 \mathrm{mM} \mathrm{NaCl}, 5 \mathrm{MgCl}_{2}$, $1 \%$ Triton $\mathrm{x}-100,1 \mathrm{mM}$ DTT, $24 \mathrm{U} / \mathrm{ml}$ Turbo DNase (Ambion). Clarified lysate was quantified and samples were boiled with 4x LDS sample (Thermo Fisher, NP0007) buffer for 5 mins at $95 \mathrm{C}$. Samples were separated on $4-12 \%$ or $12 \%$ Bolt Bis-Tris Plus Gels (Invitrogen, NP0322PK2). Proteins were transferred onto nitrocellulose membranes using Bio-Rad Trans-Blot Turbo transfer system. Membranes were blocked in Odyssey Blocking Buffer (LI-COR, 927-50000) for an hour at room temperature. Blocked membranes were incubated with primary antibody diluted in TBST and incubated overnight at $4 \mathrm{C}$ on a shaker. Primary antibodies were detected by incubating membranes 
with 1:10000 dilution of IRDye-conjugated (LI-COR) secondary anti-mouse and antirabbit antibodies for 1 hour at room temperature. Blots were visualized using LI-COR imaging system. The primary antibodies used in this study are in the reagents table.

\section{Yeast strains}

Strain BY4741 and BY4742 were used as the wild-type parental strains for the creation of the yEMC overexpression strain. Yeast homologous recombination (Rothstein, 1991) was used to generate yeast strains. For the overexpression strain, the endogenous promotor for each yEMC subunit (yEMC1, yEMC2, yEMC3, yEMC4, yEMC5, yEMC6, yEMC7, yEMC10) were replaced with a TEF2 promoter. In addition, EMC5 was tagged at the $\mathrm{C}$-terminus with linker-TEV-linker-3xFlag. Auxotrophic markers and drug selection markers in both BY4741 and BY4742 were employed to add this promoter modification to all of these eight subunits and the two strains were crossed to create the resulting BY4743 strain used for immunoprecipitation. Endogenous EMC yeast strain was made using W303a wild type parental background (leu2-3,-112; his3-11,-15; trp1-1; ura3-1; ade2-1; can1-100; MATa). Homologous recombination was used to integrate a linker-TEV-linker-3xFlag at the C-terminus of EMC coding sequence. Genomic PCR was conducted to verify integration.

\section{Design and purification of fragments antigen binding (Fab) DH4 and DE4}

Fabs were identified as described in these studies (Kim, 2011; Wu, 2012). Overexpressed yEMC solubilized in DDM as described above was biotinylated and streptavidin magnetic beads were used to capture yEMC, which was then subjected to a Fab phage library. Unbound Fabs were washed away and then binding Fabs were eluted and analyzed by ELISA. Two Fabs were identified binding EMC, Fab DH4 and DE4.

\section{Purification of DH4 and DE4 Fabs}

Plasmid with either Fab DH4 or DE4 were transformed into BL21 Gold Star cells and plated onto agarose plates with $2 \mathrm{x}$ YT $+2 \%$ glucose + Ampicillin. Cultures were inoculated from resulting colonies for overnight growth at $30{ }^{\circ} \mathrm{C}$ into $2 \mathrm{xYT}+2 \%$ glucose + Amp. In the morning dilute overnight culture to $\mathrm{OD}_{600}$ of 0.05 in $1 \mathrm{~L}$, in a $2.8 \mathrm{~L}$ flask of $2 \mathrm{xYT}+0.1 \%$ glucose + Amp. Grow the culture at $180 \mathrm{rpm}$ at $37^{\circ} \mathrm{C}$ shaker until $\mathrm{OD}_{600}$ of 0.6 , then, switch to shaking at $19^{\circ} \mathrm{C}$ for 1 hour. Next, induce with $0.4 \mathrm{mM}$ IPTG. Shake at $180 \mathrm{rpm}$ at $19{ }^{\circ} \mathrm{C}$ for $18-20$ hours. Spin $1 \mathrm{~L}$ cultures down at $3500 \mathrm{rpm}$ in large Beckman Centrifuge at $4{ }^{\circ} \mathrm{C}$ for 20 minutes in (8.1 rotor). Discard media and gently resuspend cell pellet in ice-cold $20 \mathrm{ml}$ in Buffer 1 (0.2 M Tris pH 8.0, $0.5 \mathrm{mM}$ EDTA, 0.5 $\mathrm{M}$ Sucrose) on ice. Transfer the resuspended cells from step 2 into 2 smaller JLA 25.5 centrifuge tubes. Add $20 \mathrm{ml}$ of ice cold $\mathrm{ddH}_{2} \mathrm{O}$ with $2 \mathrm{x}$ protease inhibitor cocktail (Roche Complete Ultra, Millipore Sigma 5056489001) from step 3 to the resuspended pellets. Incubate at on ice for one hour occasionally swirling samples gently. Spin periplasmic fractions at $13,000 \mathrm{x}$ g for $15 \mathrm{~min}, 4^{\circ} \mathrm{C}$, rotor 25.50. Wash 500uL Ni resin (Qiagen, NiNTA, 30210) per periplasmic fraction four times in Buffer 2 (50 mM Tris $\mathrm{pH} 8.0,250$ $\mathrm{mM} \mathrm{NaCl}$ ). Add $\mathrm{MgCl}_{2}$ and imidazole to a final concentration of $10 \mathrm{mM}$ to each 
periplasmic fraction. Add beads to periplasmic fractions and nutate at $4{ }^{\circ} \mathrm{C}$ for 2 hours. Spin down beads at $2000 \mathrm{x} \mathrm{g}, 10$ minutes, $4{ }^{\circ} \mathrm{C}$. Transfer beads either to a $50 \mathrm{~mL}$ gravity column. Wash the beads with 20 column volumes of Buffer $3(50 \mathrm{mM}$ Tris $\mathrm{pH}$ $8.0,500 \mathrm{mM} \mathrm{NaCl}, 20 \mathrm{mM}$ Imidazole). Elute protein with 3 column volumes of Buffer 4 (50 mM Tris $\mathrm{pH} 8.0,500 \mathrm{mM} \mathrm{NaCl}, 300 \mathrm{mM}$ Imidazole). Analyze eluate by SDS-PAGE 4-12\% Invitrogen (Invitrogen, NP0321PK2). Fabs as two bands run around $30 \mathrm{kDa}$ in reducing conditions, or $50 \mathrm{kDa}$ in non-reducing conditions. Dialyze eluate $\mathrm{O} / \mathrm{N}$ in Dialysis cassette $10 \mathrm{kD}$ molecular weight cutoff at $4{ }^{\circ} \mathrm{C}$ against $150 \mathrm{mM} \mathrm{KOAc}, 20 \mathrm{mM}$ HEPES pH 6.8.

\section{Purification of overexpressed yeast EMC5-3xflag}

The OE-Emc5-3xflag yeast strain were grown in YEPD media in a $40 \mathrm{~L}$ fermenter, harvested and flash frozen in liquid nitrogen. Cell pellets were thawed and diluted in lysis buffer (50 mM HEPES pH 6.8, $150 \mathrm{mM}$ KOAc, $2 \mathrm{mM} \mathrm{MgOAc}, 1 \mathrm{mM} \mathrm{CaCl} 2,0.2 \mathrm{M}$ Sorbital, $2 x$ Protease Inhibitor). Bead beating (10 times $\rightarrow 1$ minute on, 2 minutes off) was used to lyse cells. For $25 \mathrm{~g}$ of cells, $0.1 \mathrm{~mm}$ cold beads were added and lysis buffer up to the top of the $50 \mathrm{~mL}$ canister. After lysis, beads were filtered and solution centrifuged at 10,000 xg for 10 minutes. Supernatants were ultracentrifuged at 42,000 RPM (Ti 45 rotor) for 2 hours. Supernatant was discarded. Membrane pellet was combined with the lipid layer, and resuspended in lysis buffer and then a precooled dounce homogenizer was used to dounce 20 times. Membranes were aliquoted and flash frozen in liquid nitrogen. On ice, $150 \mathrm{~mL}$ of solubilization buffer $(50 \mathrm{mM}$ HEPES $\mathrm{pH}$ 6.8, $150 \mathrm{mM}$ KOAc, $2 \mathrm{mM}$ MgOAc, $1 \mathrm{mM} \mathrm{CaCl} 2,15 \%$ glycerol, 1\% b-DDM, 2x Protease Inhibitor) was added incrementally to $7.5 \mathrm{~g}$ of thawing membranes, nutated at $4^{\circ} \mathrm{C}$ for 1 hour in JA 25.5 rotor tubes, and centrifuged at 20,000 rpm for 45 minutes. Meanwhile $2.5 \mathrm{~mL}$ of $\alpha$ FLAG agarose beads (Millipore A2220) were rinsed in 50mL of low salt buffer (50 mM HEPES pH 6.8, $150 \mathrm{mM} \mathrm{KOAc).} \mathrm{Supernatant} \mathrm{was} \mathrm{added} \mathrm{to}$ $\alpha$ FLAG beads and nutated at $4^{\circ} \mathrm{C}$ for 2 hours. Resulting solution was applied over a glass column. After flowing through unbound solution, $\alpha$ FLAG beads were washed with 100 $\mathrm{mL}$ low salt buffer, $100 \mathrm{~mL}$ high salt buffer (50 mM HEPES pH 6.8, $300 \mathrm{mM} \mathrm{KOAc}$, $0.05 \% \mathrm{~b}$-DDM), and $100 \mathrm{~mL}$ low salt buffer. $\alpha$ FLAG beads were resuspended in $10 \mathrm{~mL}$ of low salt buffer and $300 \mathrm{uL}$ of TEV $(1.15 \mathrm{mg} / \mathrm{mL})$ was added and nutated overnight at $4^{\circ} \mathrm{C}$. Removed supernatant from beads by low speed spin and applied over $500 \mathrm{uL}$ of NiNTA beads equilibrated with low salt buffer to remove excess TEV. Flow through glass column and collect supernatant. Using a $100 \mathrm{kD}$ concentrator (Millipore, UFC910008) solution was concentrated to $2 \mathrm{mg} / \mathrm{mL}$. Concentrated EMC protein was applied to the Akta Explorer Superose 6 Increase column (Cytiva, 29091596) for size exclusion chromatography in the size exclusion buffer (20 mM HEPES pH 6.8, $150 \mathrm{mM}$ KOAc, $0.05 \%$ b-DDM). Fractions were evaluated by SDS-PAGE Coomassie stain and negative stain electron microscopy then EMC peak fractions were pooled and incubated with 2x molar excess of Fab, either Fab DH4 or Fab DE4, for 30 minutes on ice. Solution was applied to Akta Explorer Superose 6 Increase for size exclusion of Fab bound EMC. Resulting EMC-Fab fractions were evaluated by SDS-PAGE Coomassie stain and EMCFab peak fractions were pooled. 


\section{Purification and nanodisc reconstitution of endogenous yeast EMC5-3xflag}

Yeast was grown in rich media (YPAD) in a 65L fermenter until OD 2.6. Cell pellets were harvested and flash frozen in liquid nitrogen. Pellets were ground using three cycles in a French press. As above, the resulting solution was ultracentrifuged to separate membranes, dounced to homogenize, and flash frozen in liquid nitrogen. Thawed membranes were solubilized in 1\% b-DDM (Anatrace, D310) nutating at $4^{\circ} \mathrm{C}$ for 1 hour then centrifuged to separate solubilized membranes from the pellet. Supernatant was applied to equilibrated $\alpha$ FLAG beads, nutated at $4^{\circ} \mathrm{C}$ for 1 hour, and applied over a disposable plastic column at $4^{\circ} \mathrm{C}$. $\alpha$ FLAG beads were washed with low salt buffer and high salt buffer. Then washed with low salt buffer with b-DDM+CHS (Anatrace, CH210) $(10: 1)$ in place of b-DDM. $\alpha$ FLAG beads were then transferred to a $15 \mathrm{~mL}$ Eppendorf tube for TEC cleavage and nanodisc reconstitution.

Bio-Beads SM-2 (Bio-Rad) were prepared $400 \mathrm{uL}$ biobeads, rinsing with EtOH, and then water four times. Yeast Extract Total (Avanti Polar Lipids, 190000C-100mg) was prepared by transferring chloroform resuspended solution to a glass vial, drying the lipids into a film with nitrogen gas, drying in a vacuum desiccator overnight, and then solubilizing the lipids first in water and then in size exclusion buffer with DDM+CHS by bath sonication, aliquots stored at $-20^{\circ} \mathrm{C}$ until use. $200 \mathrm{uL}$ of TEV protease $(5 \mathrm{mg} / \mathrm{mL})$ and $150 \mathrm{uL}$ of $1 \mathrm{mg} / \mathrm{mL}$ Yeast Total Extract solubilized in b-DDM+CHS, at room temperature for 30 minutes. Then added MSP1D1, purified as described previously (Ritchie, 2009), to a ratio of 200:10:1 (Yeast total extract:MSP1D1:EMC), at $4^{\circ} \mathrm{C}$ for 10 minutes. Then activated Bio-Beads SM-2 (Bio-Rad), $300 \mathrm{uL}$, were added and nutated overnight. On-bead reconstitution employed adapted from (Laverty, 2019). In the morning $~ 100 \mathrm{uL}$ more Bio-Beads SM-2 (Bio-Rad) were added and 2x molar excess of FabDH4, nutated for another hour. Beads and solution applied to an EconoPac column (Bio-Rad). Flow through was collected and solution was applied to a $100 \mathrm{kD}$ (Amicon) concentrator. Resulting concentrated EMC was applied to the Akta Explorer Superose 6 Increase column for size exclusion chromatography. Peak fractions were pooled for SDSPAGE Coomassie stain, negative stain, and cryo-EM evaluation.

\section{Cryo-EM Sample Preparation and Data Collection for yEMC} Overexpressed EMC + Fab DE4 in b-DDM

Following size exclusion sample was prepared for cryo electron microscopy. $3 \mu \mathrm{L}$ of sample $(0.1 \mathrm{mg} / \mathrm{mL}$ EMC + Fab DE4 in 20mM HEPES pH 6.8, $150 \mathrm{mM} \mathrm{KOAc,} 0.05 \%$ bDDM) was applied to the grid, incubated for 10 seconds, then blotted with no offset for 6.5 seconds and plunge frozen in liquid ethane using a Vitrobot Mark III at $5^{\circ} \mathrm{C}$, Whatman \#1 filter paper, and 100\% humidity. Protein was frozen on glow discharged Ultrathin Carbon Film on a Lacey Carbon Support Film (Ted Pella, 01824) and stored under liquid nitrogen until imaging. This dataset was collected on the $300 \mathrm{kV}$ Technai Polara at UCSF with a $30 \mu \mathrm{m} \mathrm{C} 2$ aperture, $100 \mu \mathrm{m}$ Objective aperture, and K2 Summit detector operated in super-resolution mode. 1536 micrographs were collected using SerialEM (Mastronarde, 2005) at a magnification of $31,000 \mathrm{X}(0.6078 \AA ̊$ / super resolution 
pixel) as dose-fractionated stacks of 40 frames x 0.2 second exposures $\left(1.42 \mathrm{e}^{-} / \AA^{2}\right)$ for a total dose of $\sim 56.85 \mathrm{e}^{-/ \AA^{2}}$ (see Table S1).

\section{Overexpressed EMC + Fab DH4 in b-DDM}

Following size exclusion sample was prepared for cryo electron microscopy. $3 \mu \mathrm{L}$ of sample $(0.1 \mathrm{mg} / \mathrm{mL}$ EMC + Fab DH4 in 20mM HEPES pH 6.8, $150 \mathrm{mM} \mathrm{KOAc,} 0.05 \%$ bDDM) was applied to the grid, incubated for 10 seconds, then blotted with no offset for 7 seconds and plunge frozen in liquid ethane using a Vitrobot Mark III at $4^{\circ} \mathrm{C}$, Whatman \#1 filter paper, and $100 \%$ humidity. Protein was frozen on glow discharged Ultrathin Carbon Film on a Lacey Carbon Support Film (Ted Pella 01824). This dataset was collected at the HHMI Janelia Research Campus on Titan Krios 2, a $300 \mathrm{kV}$ microscope equipped with a $50 \mu \mathrm{m} \mathrm{C} 2$ aperture, $70 \mu \mathrm{m}$ objective aperture, and K2 Summit detector operated in super-resolution mode. 3357 micrographs were collected using automated SerialEM (Mastronarde, 2005) collection with defocus range set between -1 and $-3 \mu \mathrm{m}$ at a magnification of $22,500 \mathrm{X}(0.655 \AA$ / super resolution pixel) as dose-fractionated stacks of 50 frames x 0.2 second exposures $\left(1.165 \mathrm{e}^{-} / \AA^{2}\right)$ for a total dose of $\sim 58.3 \mathrm{e}^{-} / \AA^{2}$ (see Table S1).

Endogenous EMC + Fab DH4 in MSP1D1-Yeast Total Extract nanodisc Following size exclusion sample was prepared for cryo electron microscopy. $4 \mu \mathrm{L}$ of sample $(\sim 0.8 \mathrm{mg} / \mathrm{mL}$ EMC + Fab DH4 in nanodisc in 20mM HEPES pH 6.8, $150 \mathrm{mM}$ KOAc, $0.05 \%$ b-DDM) was applied to the grid from the left side, then blotted with no offset for 2.5 seconds, then another $4 \mathrm{uL}$ of sample was applied to the right side of the grid (without glow discharge) and blotted for 3.5 seconds, and plunge frozen in liquid ethane using a Vitrobot Mark IV at $4^{\circ} \mathrm{C}$, Whatman \#1 filter paper, and $100 \%$ humidity. Protein was frozen on R 1.2/1.3 grids with 300 Au mesh (Quantifoil, Germany). This dataset was collected at UCSF on the Titan Krios 2, a $300 \mathrm{kV}$ microscope equipped with a $70 \mu \mathrm{m} \mathrm{C} 2$ aperture, $100 \mu \mathrm{m}$ objective aperture, and K3 detector operated in CDS mode. 5949 micrographs were collected using automated SerialEM (Mastronarde, 2005) collection with defocus range set between -0.8 and $-2 \mu \mathrm{m}$ at a magnification of $105 \mathrm{X}$ $(0.4265 \AA$ / super resolution pixel) as dose-fractionated stacks of 100 frames x 0.06 second exposures $\left(0.67 \mathrm{e}^{-/} \AA^{2}\right)$ for a total dose of $\sim 67 \mathrm{e}^{-} / \AA^{2}$ (see Table S1).

\section{Image Analysis and 3D Reconstruction for yEMC \\ Overexpressed EMC + Fab in b-DDM}

Image processing schematic (Figure 2 - Figure Supplement 1) and Supplementary Table 1 have additional details. All dose-fractionated image stacks were corrected for motion artefacts, $2 \mathrm{x}$ binned in the Fourier domain, and dose-weighted using MotionCor $(\mathrm{Li}, 2013)$ for the DDM datasets, resulting in one dose-weighted and one unweighted integrated image per stack with pixel sizes of $1.22 \AA ̊$ (DDM - Polara) or 1.31 (DDM Janelia Krios). The parameters of the Contrast Transfer Function (CTF) were estimated using GCTF-v1.06 (Zhang, 2016) and the motion-corrected but unweighted images. For each dataset $\sim 1000$ particles per dataset were manually selected and averaged in 2D using RELION 2.0 (Kimanius, 2016). The resulting class sums were then used as templates for automated particle picking using Gautomatch-v0.55 (Zhang, 2016), followed by extraction in RELION 2.0. Five rounds of 2D classification were performed to eliminate 
ice contamination, particles near carbon edges, and 2D class without visible secondary structure features. Subsequent particles were subjected to 3D auto-refine in Relion 2.0. The Polara dataset was processed providing a reference model created in Spider (Shaikh, 2008 ) roughly mimicking the dimensions seen in $2 \mathrm{D}$ projections, then a second round was run using the resulting volume before two rounds of 3D classification without alignments. The resulting subset of particles were subjected to 3D auto-refine and then $3 \mathrm{D}$ classification with local alignments. The best 83,599 particles were then subjected to $3 \mathrm{D}$ refinement resulting in a 3D volume with $\sim 8 \AA$ reported resolution, which was rescaled and low-pass filtered for use as the reference for the DDM Krios dataset. 3D classification without alignments, 3D refinement, 3D classification with local alignments, and 3D auto refinement were performed resulting in a $\sim 7 \AA$ structure composed of 170,186 particles. Both resulting reconstructions overlay with one another, despite having Fab DH4 in one sample and DE4 in the other. Furthermore, they both displayed a severe orientation bias, and $3 \mathrm{D}$ reconstructions appeared streaky.

Particles from both datasets were re-extracted and scaled to a common pixel size of 1.35 $\AA$ and box size of 266 . The combined dataset was subjected to two rounds of $3 \mathrm{D}$ refinement to form a consensus structure at $\sim 6.8 \AA$ all conducted in Relion 2.0. These particles were then subjected to 3D refinement in THUNDER (Hu, 2018) using softedged mask. THUNDER produced a resulting 3D reconstruction that visually appeared less distorted along the axis of overrepresented views and resulted in a $\sim 4.8 \AA$ consensus structure. Postprocessing was done in Relion 3.0 resulting in a $\sim 4.3 \AA$ sharpened map and output was used to generate the FSC plot (Figure 2 - figure supplement 1). Molecular graphics and analyses were performed with the UCSF Chimera package (Pettersen, 2004) and Coot 0.8.7 and Coot 0.9 (Emsley, 2004; Emsley, 2010). Local resolution was computed by inputting mask and half maps into Cryosparc 2 local resolution (Stagg, 2014; Punjani, 2017; Punjani, 2019) and visualizing the resulting map and scaling in UCSF Chimera.

\section{Endogenous EMC + Fab DH4 in MSP1D1-Yeast Total Extract nanodisc}

All dose-fractionated image stacks were corrected for motion artefacts, $2 x$ binned in the Fourier domain, and dose-weighted using MotionCor2 (Zheng, 2017) using Focus (Biyani, 2017) resulting in a $2 x$ binned pixel size of $0.835 \AA$ (nanodisc - UCSF Krios). The parameters of the Contrast Transfer Function (CTF) were estimated using GCTFv1.06 (Zhang, 2016) and the motion-corrected but unweighted images. Data were then split into five groups of 1000 micrographs for processing until they were combined in $3 \mathrm{D}$. Roughly $\sim 1000$ particles per subset were manually selected and averaged in $2 \mathrm{D}$ using RELION 3.0 (Zivanov, 2018) for the nanodisc dataset. The resulting class sums were then used as templates for automated particle picking using Autopick in Relion 3.0, followed by extraction and one round of $2 \mathrm{D}$ classification per subset to remove ice contamination. The resulting subsets of particles were subject to 3D refinement. Combining the RELION star files these particles were imported into Cryosparc 2.0 (Punjani, 2017; Punjani, 2019) along with a reference model. These data were subjected to non-uniform homogenous refinement, a round of four class $3 \mathrm{D}$ heterogeneous refinement, another round of non-uniform refinement for the best class (roughly 1.2 million particles), non-uniform homogeneous refinement, a round of two class $3 \mathrm{D}$ 
heterogeneous refinement, and another non-uniform homogeneous refinement for the best class (roughly 500,000 particles). These were then exported to RELION 3.0 using PyEM (Asarnow, 2019). 3D Classification was performed with local alignments, then CTF refinement of the best class (230,528 particles) resulting in a $\sim 3.2 \AA$ final reconstruction. This was post-processed in both RELION 3.0 and using phenix.autosharpen, both resulting maps were used for model building.

\section{Model building and refinement of yEMC in nanodiscs}

Structural biology applications used in this project were compiled and configured by SBGrid (Morin, 2013). The yeast EMC structure was built de novo using Coot (version 0.8.7 and 0.9) and UCSF ChimeraX (Goddard, 2018). Visible secondary structure was built by hand for the entire structure using overlays of the yEMC detergent consensus map as well as the yEMC nanodisc unsharpened and sharpened map. Starting with the best resolved transmembrane helices, sequence was placed for each of the predicted transmembrane helices, using TMHMM (Krogh, 2001), in the yEMC proteins. Visual inspection for landmark residues (tryptophan, tyrosine, leucine, and proline) in the sequences that correlated with the position of well densities as well as fit correlation in UCSF Chimera was computed to assign identities for yEMC1, yEMC3, yEMC5, and yEMC6. Connectivity between the EMC1 assigned helix to the lumenal domain was used to start assigning sequence for the lumenal portion of EMC1. Secondary structure prediction was computed for all yEMC proteins using Phyre2 (Kelley, 2015) and Quick2D, a tool within the Max-Plank Institute for Developmental Biology Bioinformatics Toolkit that visualizes several different secondary structure predictors (Jones, 1999; Cuff and Barton, 1999; Ouali and King, 2000; Rost, 2001; Lupas, 1991; Jones, 1994; Ward, 2004; Peng, 2006; Obradovic, 2005). Secondary structure prediction was used to check and guide sequence assignment of beta strands and helices. Next several homology models were computed and overlain for yEMC2, with a predicted TPR structural domain, using Robetta (Raman, 2009; Song, 2013), I-TASSER (Zhang, 2008; Roy, 2010; Yang, 2015), Phyre2 (Kelley, 2015), and RaptorX (Kallberg, 2012). These were used in addition to secondary structure prediction to guide sequence assignment, loop building, and helical packing. Fab DH4 starting structure was computed using Phyre 2 1-to-1 threading against a crystal structure of a monoclonal Fab (PDB 1M71, Vyas, 2002). EMC3, EMC5, and EMC6 were built off of the transmembrane helices using sphere refinement, real space refinement, regularization, and visual monitoring of the Ramachandran plot in Coot. EMC7 and EMC10 both form beta sandwich folds on the exterior of the EMC1 lumenal domain, beta strand sequence was placed for both in both densities, position of aromatic residues and loop length differed between the two allowing assignment of each. After building EMC1-3, EMC5-7, and EMC10, there remained several transmembrane helices and a beta strand fitted into the lumen but not connected to EMC1, EMC7, or EMC10. The resolution of the lumenal domain is better than $3 \AA$ in most parts allowing for sequence placement of the EMC4 C-terminus and $\mathrm{C}$-terminal transmembrane helix. The connectivity of the transmembrane helix to the cytoplasmic domain was not resolved. However, there was an additional poorly resolved short helix and loop density in the cytoplasmic domain which was assigned to EMC4. 
Two poorly resolved transmembrane helices remained, however due to the fact they did not have clear connectivity to any built strand, poly alanine alpha helices were built in but not assigned to a yEMC protein (Figure 5 - figure supplement 3). EMC4 had density in the cytoplasmic domain as well as the lumenal domain, suggesting that it has either one or three transmembrane passes. EMC7 and EMC10 were predicted to have transmembrane helices however the connection between the lumenal densities and those predicted transmembrane helices was not clear. Additional density that was not built into was visualized in UCSF ChimeraX (Goddard, 2018) and allowed for subsequent assignment of several glycosylated residues and one POPC molecule. Each subunit was built in a separate pdb file and subjected to iterative rounds of phenix.real_space_refine (Adams, 2011; Liebschner, 2019) into segmented maps preceded and followed by adjustment in Coot. Manual assignment of secondary structure restraints was used and improved during Phenix refinement. Once all of the well resolved secondary structure was assigned to yEMC subunits, PDBs were combined and subjected to iterative rounds of phenix.real_space_refine (Adams, 2011; Afonine, 2018; Liebschner, 2019) in the unsharpened and then sharpened maps. Loops were built back where the connectivity was clear and then refined again in Phenix and Coot. PDBs were prepared for refinement steps using phenix.reduce to add hydrogens throughout refinement steps, ReadySet to generate cif restraints, and Phenix PDB preparation tool for creating mmCIF files for deposition. Representative regions of the model as well as the map-to-model FSC can be found in Figure 2 - Figure Supplement 5.

\section{Cryo-EM sample preparation and imaging for hEMC}

$4 \mu \mathrm{L}$ of freshly purified hEMC (in detergent or nanodisc) was applied to glow discharged copper Quantifoil holey carbon grids (R1.2/1.3 $300 \mathrm{mesh}$ ) at $100 \%$ humidity and $4{ }^{\circ} \mathrm{C}$ in a Vitrobot Mark IV (Thermo) and incubated for 30 seconds. Excess liquid was blotted away with filter paper (blot force $4-6$, blot time 4 seconds) and the grid plunge-frozen into liquid ethane. Samples were imaged on a FEI Titan Krios microscope operating at $300 \mathrm{kV}$, equipped with a post-column GIF and a K3 direct detector operating in counting mode. Images were recorded at a nominal magnification of $105,000 \mathrm{x}(0.8512 \AA /$ pixel at the specimen level) for hEMC in nanodiscs or $81,000 \mathrm{x}(1.094 \AA /$ pixel at the specimen level) for hEMC in detergent, with target defocus ranging between 0.7 and $2.8 \mu \mathrm{m}$ and total exposure of $\sim 70 \mathrm{e} / \AA^{2}$ using SerialEM (Mastronarde, 2005). On-the-fly motion correction, CTF estimation and templated particle auto-picking were performed using a pipeline implemented in Focus (Biyani, 2017).

\section{Cloning and expression constructs for hEMC}

A modified version of the biGBac (Weissmann, 2016) multi-gene cloning method was combined with the BacMam (Goehring, 2014) mammalian expression system to allow for recombinant production of human EMC (hEMC). hEMC subunits were individually inserted into pEG, with EMC5 bearing a C-terminal Flag-tag. To amplify geneexpression cassettes (GEC) from $\mathrm{pEG}$, original forward primers from biGBac were used in combination with modified reverse primers bearing complementarity downstream of 
the SV40 terminator sequence. GECs were inserted into pBIG1a-e vectors as follows: pBIG1a (EMC1 - Uniprot code Q8N766-1), pBIG1b (EMC4 - Q5J8M3-1; EMC5-Flag Q8N4V1-1, which encodes DYKDDDDK immediately after R131; EMC6 - Q9BV81), pBIG1c (EMC2 - Q15006; EMC3 - Q9P0I2-1; EMC7 - Q9NPA0), pBIG1d (EMC8 O43402-1; EMC9 - Q9Y3B6), pBIG1e (EMC10 - Q5UCC4-1). These were subsequently combined into $\mathrm{pBIG} 2 \mathrm{abcde}$ to yield a single expression vector containing all ten hEMC subunits. Bacmid was generated in DH10 EMBacY E. coli and subsequently transfected into Sf9 insect cells using FuGENE (Promega) reagent. Virus was amplified in $\mathrm{Sf} 9$ cells up to $\mathrm{P} 3$ and virus supernatant sterilized by filtration.

\section{hEMC expression, purification and nanodisc reconstitution}

Recombinant hEMC was expressed by baculovirus transduction of human embryonic kidney (HEK) 293S GnTI- cells grown in suspension. Cells were maintained at $37^{\circ} \mathrm{C}$ in Freestyle 293 Expression Medium (Thermo) and expanded with home-made suspension medium (Chaudhary, 2012) in $2 \mathrm{~L}$ shaker flasks. For expression of hEMC, 10\% (v/v) P3 virus was added to $800 \mathrm{~mL}$ of HEK culture at a cell density $>3 \times 10^{6} .16$ hours posttransduction, $10 \mathrm{mM}$ butyrate was added and the temperature reduced to $30^{\circ} \mathrm{C}$. Cells were harvested 48 hours later and stored frozen at $-80{ }^{\circ} \mathrm{C}$.

For purification, $15-20 \mathrm{~g}$ of cell pellet was thawed and resuspended in $60-80 \mathrm{~mL}$ Lysis Buffer containing $50 \mathrm{mM}$ ammonium citrate $\mathrm{pH} 6.0,150 \mathrm{mM}$ sodium chloride, 0.001 $\mathrm{mg} / \mathrm{mL}$ Benzonase, EDTA-free protease inhibitor cocktail (1 tablet per $50 \mathrm{~mL}$ of buffer), and lysed by Dounce homogenization on ice (50 strokes). Glyco-diosgenin (GDN, Anatrace) was added to the lysate at $2 \%(\mathrm{w} / \mathrm{v})$ and cellular membranes solubilized for 3 hours at $4{ }^{\circ} \mathrm{C}$ under constant stirring. Insolubilized material was removed by centrifugation at $100,000 \mathrm{x}$ g, supernatant incubated with $2 \mathrm{~mL}$ M2 Flag-affinity resin inbatch for 2 hours at $4{ }^{\circ} \mathrm{C}$. The resin was poured into a column and unbound proteins washed away with 25 column volumes (CV) of Wash Buffer containing $20 \mathrm{mM}$ ammonium citrate $\mathrm{pH}$ 6.0, $150 \mathrm{mM}$ sodium chloride, 0.01\% (w/v) GDN. Bound hEMC was eluted in $10 \mathrm{CV}$ Wash Buffer containing $0.3 \mathrm{mg} / \mathrm{mL}$ Flag peptide and concentrated to $<500 \mu \mathrm{L}$ using centrifugal concentration filters with $100 \mathrm{kDa}$ cut—off (Amicon). Sample was polished using size-exclusion chromatography (SEC) on a Superose 6 Increase 10/300 GL column (GE Healthcare) with Running Buffer containing $10 \mathrm{mM}$ ammonium citrate $\mathrm{pH}$ 6.0, $100 \mathrm{mM}$ sodium chloride, $0.25 \mathrm{mM}$ TCEP, $0.01 \%$ (w/v) GDN. Peak fractions containing hEMC were pooled, concentrated to $\sim 3 \mathrm{mg} / \mathrm{mL}$ and used immediately for cryo-EM grid preparation.

hEMC in nanodiscs composed of MSP1D1 scaffold protein and 1-palmitoyl-2-oleoyl-snglycero-3-phosphatidylcholine (POPC) was reconstituted following Flag-affinity chromatography. The MSP1D1 expression vector was a gift from Franz Hagn (TUM, Germany) and the scaffold protein purified from E. coli following a published protocol (Hagn, 2018). Prior to reconstitution, hEMC purified by Flag-affinity chromatography was mixed with MSP1D1 and POPC (solubilized as $25 \mathrm{mM}$ stock in 5\% n-dodecyl $\beta$-Dmaltoside) in a 1:4:50 ratio and this mixture incubated on ice for 2 hours. Nanodisc reconstitution was achieved by incubation with $0.5-1 \mathrm{~mL}$ Bio-Beads SM-2 (Bio-Rad) for 16 hours at $4^{\circ} \mathrm{C}$ under constant rotation. The liquid phase was aspirated, concentrated 
to $<500 \mu \mathrm{L}$ and injected onto a Superose 6 SEC column with buffer containing $10 \mathrm{mM}$ ammonium citrate $\mathrm{pH}$ 6.0, $100 \mathrm{mM}$ sodium chloride, $0.25 \mathrm{mM}$ TCEP, to separate nanodisc-embedded hEMC from empty nanodiscs. Peak fractions were pooled and concentrated to $\sim 2 \mathrm{mg} / \mathrm{mL}$ for immediate cryo-EM grid preparation.

\section{Cryo-EM sample preparation and imaging for hEMC}

$4 \mu \mathrm{L}$ of freshly purified hEMC (in detergent or nanodisc) was applied to glow discharged copper Quantifoil holey carbon grids (R1.2/1.3 $300 \mathrm{mesh}$ ) at $100 \%$ humidity and $4{ }^{\circ} \mathrm{C}$ in a Vitrobot Mark IV (Thermo) and incubated for 30 seconds. Excess liquid was blotted away with filter paper (blot force $4-6$, blot time 4 seconds) and the grid plunge-frozen into liquid ethane. Samples were imaged on a FEI Titan Krios microscope operating at $300 \mathrm{kV}$, equipped with a post-column GIF and a K3 direct detector operating in counting mode. Images were recorded at a nominal magnification of $105,000 \mathrm{x}(0.8512 \AA / \mathrm{pixel}$ at the specimen level) for hEMC in nanodiscs or $81,000 \mathrm{x}(1.094 \AA /$ pixel at the specimen level) for hEMC in detergent, with target defocus ranging between 0.7 and $2.8 \mu \mathrm{m}$ and total exposure of $\sim 70 \mathrm{e} / \AA^{2}$ using SerialEM (Mastronarde, 2005). On-the-fly motion correction, CTF estimation and templated particle auto-picking were performed using a pipeline implemented in Focus (Biyani, 2017).

\section{Cryo-EM data processing for hEMC in detergent}

Preprocessing in Focus included dose-weighted motion correction using Motioncor2 (Zheng, 2017), CTF estimation using Gctf (Zhang, 2016) and templated autopicking using Gautomatch (Kai Zhang). The autopicking template originated from a reconstruction of hEMC in GDN micelles, with data acquired on a K2 (Gatan) direct electron detector (operated in counting mode) under liquid nitrogen conditions using a Glacios microscope (Thermo) operated at $200 \mathrm{kV} .3713$ micrographs with a maximal resolution estimate better than $5 \AA$ were imported into Relion 3.0 (Zivanov, 2018), from which $\sim 3.35$ million particles were extracted applying 4-fold binning. These were subjected to three rounds of 2D classification and two rounds of 3D classification (using the reconstruction obtained from the $200 \mathrm{kV}$ dataset as reference), followed by $3 \mathrm{D}$ autorefinement. This reconstruction was used as initial model for three rounds of 3D classification of the original $\sim 3.35$ million particles (first round: $\mathrm{K}=10, \mathrm{~T}=10$; second round: $\mathrm{K}=10, \mathrm{~T}=10$; third round: $\mathrm{K}=3, \mathrm{~T}=16$ ), yielding a set of 144,222 particles. This set was re-extracted at full pixel size, followed by masked 3D autorefinement, producing a reconstruction at $3.77 \AA$ overall resolution. Application of non-uniform refinement in cryoSPARC (Punjani, 2017; Punjani, 2019) further improved the map quality and overall resolution to $3.60 \AA$.

\section{Cryo-EM data processing for hEMC in nanodiscs}

Micrographs were preprocessed using Focus in a similar manner as for hEMC in detergent. 9164 micrographs with a maximal resolution estimate better than $5 \AA$ were imported into Relion 3.0, from which $\sim 5.9$ million particles were extracted applying 4fold binning. These were subjected to three rounds of $3 \mathrm{D}$ classification (using hEMC in GDN as reference for the first round), after which 386739 particles were kept and re- 
extracted to full pixel size. Particles were aligned using global angular search 3D classification $(\mathrm{K}=1, \mathrm{~T}=4)$ before one further round of $3 \mathrm{D}$ classification with a soft mask and skipping alignment $(\mathrm{K}=6, \mathrm{~T}=8)$, to isolate a set of 177560 homogeneous hEMC particles. Masked 3D autorefinement of this particle set yielded a map at $3.6 \AA$ overall resolution. Implementation of cryoSPARC non-uniform refinement led to a consensus map at $3.4 \AA$ global resolution. To aid de novo model building of cytoplasmic and luminal domains, these parts were subjected to masked focused classification $(K=5$, $\mathrm{T}=8$ ), 3D autorefinement and post-processing in Relion, yielding improved maps at 3.4 $\AA$ and $3.2 \AA$, respectively. To obtain highest quality maps of the transmembrane domains, the 177560 particles from consensus refinement were processed using Sidesplitter (Ramlaul, 2020), producing a $3.3 \AA$ global map after Relion post-processing, where transmembrane helix pitch and side chains were well resolved and allowed for unambiguous sequence assignment. The final particle set was further subjected to 3D variability analysis (Punjani, 2020) in cryoSPARC, revealing the presence or absence of the EMC7 lumenal domain between the EMC1 beta-propellers. Heterogeneous refinement, using a map from 3D variability analysis containing stronger EMC7 density as reference, allowed for further sub-classification of the consensus particle set. Nonuniform refinement of the class containing stronger EMC7 density produced a map at 3.5 $\AA$ global resolution, which was subsequently used to build an EMC7 model.

\section{Model building and refinement of hEMC in nanodiscs and detergent}

Given the higher quality hEMC nanodisc map compared to the detergent map, the former was used for de novo model building in Coot (Emsley, 2004; Emsley, 2010). Focused luminal and cytoplasmic, as well as Sideplitter maps, permitted assignment of amino acid sequence throughout all parts of hEMC. Inspection of structural homology and secondary structure predictions for the hEMC subunits produced via HHpred and Quick2D servers (Zimmermann, 2018) predicted the luminal domain of EMC1, the largest hEMC subunit, to consist of two beta-propellers. EMC7 and EMC10 are predicted to feature betasandwich structures in the lumen. A final missing beta-strand of the EMC1 membrane proximal propeller could be assigned to the luminal C-terminus of EMC4, which forms a parallel sheet with EMC1 residues $668-674$. Almost all of EMC2 is predicted to form an alpha-solenoid structure harboring several TPR motifs. Analysis of EMC8 and EMC9 amino acid sequences revealed structural homology to CSN5 (deneddylase subunit of the CSN complex) and Rpn11 (deubiquitinase subunit of the 19S proteasomal regulatory particle) peptide hydrolase folds. The globular density sitting on the distal face of the EMC2 solenoid, facing away from the rest of the complex, was modeled with the EMC8 sequence, which shares $\sim 45 \%$ amino acid sequence identity with EMC9. Additional helical density sitting sideways on top of the EMC2 solenoid could be modeled as two cytoplasmic helices of EMC3 as well as the extended, partially helical meander of the EMC3 C-terminus. Beta-strand-like density on the EMC8 surface, commonly occupied by deubiquitinase substrate peptides, was assigned to the extreme N-terminus of EMC4, with a further downstream part of this cytoplasmic domain snaking along EMC2 and EMC3 towards the transmembrane part of hEMC.

Clear side-chain resolution and excellent connectivity of the Sidesplitter map, within the nanodisc encircled membrane domain, allowed us to model all predicted transmembrane 
helices of EMC1, EMC3, EMC5 and EMC6. EMC5 extends its C-terminus outside the membrane, which snakes through the central cavity of the EMC2 solenoid on the cytoplasmic side. Inspection of the map at lower thresholds revealed density for at least two additional transmembrane helices facing EMC3 and EMC6 on one side of the complex: continuous density from one of these helices towards the luminal EMC4 Cterminus indicates that at least one of these gate helices represent EMC4's C-terminal transmembrane helix. However, given poor map resolution and connectivity in this region, we left the other gate helices unassigned.

Model refinement was performed using real-space refinement in Phenix (Adams, 2011), applying secondary structure and Ramachandran restraints. Initially, luminal and cytoplasmic domains were refined individually against their focused maps, after which the improved models were rigid-body placed and refined against the non-uniform refined consensus map. The transmembrane domain was likewise first refined against the Sidesplitter map, after which all parts of hEMC were combined into a consensus model and refined against the consensus map.

The refined $\mathrm{hEMC}$ nanodisc model was subsequently docked into the hEMC detergent map, revealing a relative rotation of the entire lumenal domain. The fitted model was manually adjusted in Coot (Emsley, 2004; Emsley, 2010) and refined using Phenix realspace refinement (Adams, 2011). Different masking strategies failed to produce stronger density for the EMC7 lumenal domain in the hEMC detergent maps, despite EMC7 levels being comparable to the other hEMC subunits in subsequent mass spectrometry analysis. EMC7 thus remains absent from our hEMC detergent model, perhaps due to conformational heterogeneity.

\section{Mass spectrometric analysis of purified hEMC samples in detergent or nanodiscs}

GDN solubilized or nanodisc reconstituted hEMC purified by Flag-affinity chromatography and SEC was subjected to mass spectrometric analysis to assess hEMC subunit abundance. For reduction and alkylation of the proteins, proteins were incubated with SDC buffer (1\% Sodiumdeoxycholate, 40nmM 2-Cloroacetamide (SigmaAldrich), $10 \mathrm{mM}$ tris(2-carboxyethyl) phosphine (TCEP; PierceTM, Thermo Fisher Scientific) in $100 \mathrm{mM}$ Tris, $\mathrm{pH}$ 8.0) for $20 \mathrm{~min}$ at $37^{\circ} \mathrm{C}$. Before digestion the samples were diluted 1:2 with MS grade water (VWR). Samples were digested overnight at $37^{\circ} \mathrm{C}$ with $1 \mu \mathrm{g}$ trypsin (Promega).

The solution of peptides was then acidified with Trifluoroacetic acid (Merck) to a final concentration of $1 \%$ and a $\mathrm{pH}$ value of $<2$, followed by purification via SCX StageTips (Rappsilber, 2007) washed with 1\% TFA in Isopropanol, followed by a second wash with $0.2 \%$ TFA, eluted as one fraction with $80 \%$ Acetonitrile and 5\% Ammonia (Merck). Samples were vacuum dried and re-suspended in $6 \mu 1$ of Buffer A $(0.1 \%$ Formic acid (Roth) in MS grade water (VWR)).

Purified and desalted peptides were loaded onto a 15-cm column (inner diameter: 75 microns; packed in-house with ReproSil-Pur C18-AQ 1.9-micron beads, Dr. Maisch $\mathrm{GmbH}$ ) via the autosampler of the Thermo Easy-nLC 1000 (Thermo Fisher Scientific) at 
$50{ }^{\circ} \mathrm{C}$. Using the nanoelectrospray interface, eluting peptides were directly sprayed onto the benchtop Orbitrap mass spectrometer Q Exactive HF (Thermo Fisher Scientific). Peptides were loaded in buffer A $(0.1 \%$ (v/v) Formic acid) at $250 \mathrm{nl} / \mathrm{min}$ and percentage of buffer B ( $80 \%$ Acetonitril, $0.1 \%$ Formic acid) was ramped to $30 \%$ over 45 minutes followed by a ramp to $60 \%$ over 5 minutes then $95 \%$ over the next 5 minutes and maintained at $95 \%$ for another 5 minutes. The mass spectrometer was operated in a datadependent mode with survey scans from 300 to $1650 \mathrm{~m} / \mathrm{z}$ (resolution of 60000 at $\mathrm{m} / \mathrm{z}$ $=200$ ), and up to 10 of the top precursors were selected and fragmented using higher energy collisional dissociation (HCD with a normalized collision energy of value of 28). The MS2 spectra were recorded at a resolution of $15000($ at $\mathrm{m} / \mathrm{z}=200)$. AGC target for MS and MS2 scans were set to 3E6 and 1E5 respectively within a maximum injection time of 100 and $60 \mathrm{~ms}$ for MS and MS2 scans respectively. Dynamic exclusion was set to $30 \mathrm{~ms}$.

Raw data were processed using the MaxQuant computational platform (Cox, 2008) with standard settings applied. Shortly, the peak list was searched against the reviewed human Uniprot database with an allowed precursor mass deviation of $4.5 \mathrm{ppm}$ and an allowed fragment mass deviation of $20 \mathrm{ppm}$. MaxQuant by default enables individual peptide mass tolerances, which was used in the search. Cysteine carbamidomethylation was set as static modification, and methionine oxidation and $\mathrm{N}$-terminal acetylation as variable modifications. The iBAQ algorithm was used for calculation of approximate abundances for the identified proteins (Schwanhäusser, 2011) which normalizes the summed peptide intensities by the number of theoretically observable peptides of the protein.

\section{Sequence alignments}

T-coffee PSI-Coffee extension (Notredame, 2000) was used to compute sequence alignments between yEMC, hEMC, and homologous proteins (Figure 1 - figure supplement 6-7, Figure 3 - figure supplement 3, Figure 5 - figure supplement 4). Outputs of these alignments were visualized in Jalview (Waterhouse, 2009) for figure creation and colored by ClustalX convention.

\section{Figure and video creation}

All figures were assembled and edited in Adobe Illustrator. Figure 1 and Figure 1 figure supplement 3 were created using BioRender. All of the visualization, structure figures, and structure videos were made using UCSF ChimeraX 1.0 (Goddard, 2018) and UCSF Chimera 1.14 (Pettersen, 2004). Flow cytometry plots were generated in Python and labeled in Adobe Illustrator.

\section{Acknowledgements}

We thank J. Weibazahn, P. Walter, R. Irannejad, J. Gestwicki, R. Scheltema, Ö. Karayel, H. Nguyen, I. Johnson, N. Talledge, L. Kenner, E. Thompson, K. Hickey, J. Kellermann, S. von Gronau, M. Feige, K. Swain, M. Liao, C-W. Lee, F. Wilfling, and members of the Weissman, Frost, and Schulman laboratories for assistance and helpful 
discussion; L. Metzger, Z Roe-Zurz, M. Tessema, D.W. Chester, and S. Aller for assisting with fermentation; M. Sun, H. Autzen, and E. Green for advice on nanodisc reconstitution; F. Hagn and I. Goba for the gift of the MSP1D1 vector and advice on nanodisc reconstitution; P. Thomas and D. Asarnow for computational support; $\mathrm{M}$. Braunfeld, G. Gilbert, E. Tse, D. Bulkley, M. Harrington, A. Myasnikov and Z. Yu of the UCSF Center for Advanced CryoEM for microscopy support and funded by NIH grants S10OD020054 and 1S10OD021741; J. Baker-LePain and the QB3 shared cluster (NIH grant 1S10OD021596-01) for computational support; and the Howard Hughes Medical Institute (HHMI); Z. Yu and H. Chou of the CryoEM Facility at the HHMI Janelia Research Campus (NIH grant 1S10OD021596-01). D. Bollschweiler, T. Schäfer and the cryo-EM facility at the Max Planck Institute of Biochemistry; B. Steigenberger, the mass spectrometry core facility at the Max Planck Institute of Biochemistry; E. Gouaux for the gift of the $\mathrm{pEG}$ vector; A Titan X Pascal used for this research was donated by the NVIDIA Corporation. This study was supported in part by the HDFCCC Laboratory for Cell Analysis Shared Resource Facility through a grant from NIH (P30CA082103). Molecular graphics and analyses performed with UCSF ChimeraX, developed by the Resource for Biocomputing, Visualization, and Informatics at the University of California, San Francisco, with support from National Institutes of Health R01-GM129325 and the Office of Cyber Infrastructure and Computational Biology, National Institute of Allergy and Infectious Diseases. UCSF Chimera is developed by the Resource for Biocomputing, Visualization, and Informatics at the University of California, San Francisco (supported by NIGMS P41-GM103311).

Competing interests: No competing interests.

Data and materials availability: All data needed to evaluate the conclusions in the paper are present in the paper and/or the supplementary materials, and the structural data will be made available in EMPIAR, PDB, and EMDB public databases upon publication. All of the raw data files will be in the supplemental data files and code for evaluating will available at GITHUB (MMMMMMM). Accession numbers are as follows: hEMC nanodisc map (EMD____ and model (PDB-__ _ ) hEMC detergent map (EMDand model (PDB-__ $)$; yEMC nanodisc map (EMDyEMC detergent map (EMD-

Funding: Supported by the Peter und Traudl Engelhorn-Stiftung (B.B.); the Howard Hughes Medical Institute (J.S.W.), the Chan Zuckerberg Biohub and HHMI faculty scholar fund (A.F.), and by the Leibniz prize of the Deutsche Forschungsgemeinschaft (DFG, German Research Foundation) - SCHU 3196/1-1 and the Max Planck Society (B.A.S.). M.J.S. is a Howard Hughes Medical Institute Fellow of the Helen Hay Whitney Foundation. N.T.S. was funded by the Jane Coffin Childs Memorial Fund for Medical Research (Postdoctoral Fellowship). This work was additionally supported by National Institutes of Health P50AI150476 (N.S. and C.S.C.), 1P41CA196276-01 (N.S. and C.S.C.), NIH R01 GM24485 (R.M.S.), and 1DP2OD017690-01 (A.F.). 
Author contributions: Conception and design, analysis and interpretation of data: L.E.M.V., B.B., K.D.P, N.T.S.O, J.L.B., J.R.P., E.A.B., N.S., M.J.S., R.M.S., C.S.C, B.A.S, A.F., and J.S.W.; acquisition of data: L.E.M.V., B.B., K.D.P., N.T.S.O., J.L.B., J.R.P., E.B., and N.S.; writing (original draft): L.E.M.V., B.B., and K.D.P.; writing (review and editing): L.E.M.V., B.B., K.D.P, N.T.S.O, J.L.B., J.R.P., E.B., N.S., M.J.S., R.M.S., C.S.C, B.A.S, A.F., and J.S.W

\section{References}

1. Abu-Safieh, L., Alrashed, M., Anazi, S., Alkuraya, H., Khan, A.O., Al-Owain, M., AlZahrani, J., Al-Abdi, L., Hashem, M., Al-Tarimi, S., Sebai, M.-A., Shamia, A., RayZack, M.D., Nassan, M., Al-Hassnan, Z.N., Rahbeeni, Z., Waheeb, S., Alkharashi, A., Abboud, E., Al-Hazzaa, S.A.F., Alkuraya, F.S., 2012. Autozygome-guided exome sequencing in retinal dystrophy patients reveals pathogenetic mutations and novel candidate disease genes. Genome Res 23, 236-47. https://doi.org/10.1101/gr.144105.112

2. Adams, P.D., Afonine, P.V., Bunkóczi, G., Chen, V.B., Echols, N., Headd, J.J., Hung, L.W., Jain, S., Kapral, G.J., Kunstleve, R.W.G., McCoy, A.J., Moriarty, N.W., Oeffner, R.D., Read, R.J., Richardson, D.C., Richardson, J.S., Terwilliger, T.C., Zwart, P.H., 2011. The Phenix software for automated determination of macromolecular structures. Methods 55, 94-106. https://doi.org/10.1016/j.ymeth.2011.07.005

3. Afonine, P.V., Poon, B.K., Read, R.J., Sobolev, O.V., Terwilliger, T.C., Urzhumtsev, A., Adams, P.D., 2018. Real-space refinement in PHENIX for cryo-EM and crystallography. Acta Crystallogr Sect D Struct Biology 74, 531-544. https://doi.org/10.1107/s2059798318006551

4. Amberger JS, Bocchini CA, Scott AF, Hamosh A. OMIM.org: leveraging knowledge across phenotype-gene relationships. Nucleic Acids Res. 2019 Jan 8;47(D1):D1038D1043. doi:10.1093/nar/gky1151.

5. Anghel, S.A., McGilvray, P.T., Hegde, R.S., Keenan, R.J., 2017. Identification of Oxa1 Homologs Operating in the Eukaryotic Endoplasmic Reticulum. Cell Reports 21, 37083716. https://doi.org/10.1016/j.celrep.2017.12.006

6. Asarnow, D., Palovcak, E., Cheng, Y. UCSF pyem v0.5. 2019.

Zenodo https://doi.org/10.5281/zenodo.3576630

7. Assimon, V.A., Southworth, D.R., Gestwicki, J.E., 2015. Specific Binding of Tetratricopeptide Repeat Proteins to Heat Shock Protein 70 (Hsp70) and Heat Shock Protein 90 (Hsp90) Is Regulated by Affinity and Phosphorylation. Biochemistry-us 54, 7120-31. https://doi.org/10.1021/acs.biochem.5b00801

8. Bai, L., You, Q., Feng, X., Kovach, A., Li, H., 2020. Structure of the ER membrane complex, a transmembrane-domain insertase. Nature 1-4. https://doi.org/10.1038/s41586-020-2389-3

9. Bagchi, P., Inoue, T., Tsai, B., 2016. EMC1-dependent stabilization drives membrane penetration of a partially destabilized non-enveloped virus. Elife 5, e21470. https://doi.org/10.7554/elife.21470

10. Bircham, P.W., Maass, D.R., Roberts, C.A., Kiew, P.Y., Low, Y.S., Yegambaram, M., Matthews, J., Jack, C.A., Atkinson, P.H., 2011. Secretory pathway genes assessed by high-throughput microscopy and synthetic genetic array analysis. Mol Biosyst 7, 2589. https://doi.org/10.1039/c1mb05175j

11. Biyani, N., Righetto, R.D., McLeod, R., Caujolle-Bert, D., Castano-Diez, D., Goldie, K.N., Stahlberg, H., 2017. Focus: The interface between data collection and data processing in cryo-EM. J Struct Biol 198, 124-133. https://doi.org/10.1016/j.jsb.2017.03.007 
12. Blatch, G.L., Lässle, M., 1999. The tetratricopeptide repeat: a structural motif mediating protein-protein interactions. Bioessays 21, 932-939. https://doi.org/10.1002/(sici)15211878(199911)21:11<932::aid-bies5>3.0.co;2-n

13. Borowska, M.T., Dominik, P.K., Anghel, S.A., Kossiakoff, A.A., Keenan, R.J., 2015. A YidC-like Protein in the Archaeal Plasma Membrane. Structure 23, 1715-1724. https://doi.org/10.1016/j.str.2015.06.025

14. Chaudhary, S., Pak, J.E., Gruswitz, F., Sharma, V., Stroud, R.M., 2012. Overexpressing human membrane proteins in stably transfected and clonal human embryonic kidney 293S cells. Nat Protoc 7, 453-466. https://doi.org/10.1038/nprot.2011.453

15. Chitwood, P.J., Juszkiewicz, S., Guna, A., Shao, S., Hegde, R.S., 2018. EMC Is Required to Initiate Accurate Membrane Protein Topogenesis. Cell 175, 1507-1519.e16. https://doi.org/10.1016/j.cell.2018.10.009

16. Cho, H., Stanzione, F., Oak, A., Kim, G.H., Yerneni, S., Qi, L., Sum, A.K., Chan, C., 2019. Intrinsic Structural Features of the Human IRE1 $\alpha$ Transmembrane Domain Sense Membrane Lipid Saturation. Cell Reports 27, 307-320.e5. https://doi.org/10.1016/j.celrep.2019.03.017

17. Christianson, J.C., Olzmann, J.A., Shaler, T.A., Sowa, M.E., Bennett, E.J., Richter, C.M., Tyler, R.E., Greenblatt, E.J., Harper, J.W., Kopito, R.R., 2012. Defining human ERAD networks through an integrative mapping strategy. Nat Cell Biol 14, 93-105. https://doi.org/10.1038/ncb2383

18. Coelho, J.P.L., Stahl, M., Bloemeke, N., Meighen-Berger, K., Alvira, C.P., Zhang, Z.-R., Sieber, S.A., Feige, M.J., 2019. A network of chaperones prevents and detects failures in membrane protein lipid bilayer integration. Nat Commun 10, 672 . https://doi.org/10.1038/s41467-019-08632-0

19. Cornell, C.E., Mileant, A., Thakkar, N., Lee, K.K., Keller, S.L., 2020. Direct imaging of liquid domains in membranes by cryo-electron tomography. Proc National Acad Sci 202002245. https://doi.org/10.1073/pnas.2002245117

20. Corradi, V., Sejdiu, B.I., Mesa-Galloso, H., Abdizadeh, H., Noskov, S.Y., Marrink, S.J., Tieleman, D.P., 2019. Emerging Diversity in Lipid-Protein Interactions. Chem Rev 119, 5775-5848. https://doi.org/10.1021/acs.chemrev.8b00451

21. Costa, E.A., Subramanian, K., Nunnari, J., Weissman, J.S., 2018. Defining the physiological role of SRP in protein-targeting efficiency and specificity. Sci New York N Y 359, 689-692. https://doi.org/10.1126/science.aar3607

22. Cox, J., Mann, M., 2008. MaxQuant enables high peptide identification rates, individualized p.p.b.-range mass accuracies and proteome-wide protein quantification. Nat Biotechnol 26, 1367-1372. https://doi.org/10.1038/nbt.1511

23. Cuff, J.A., Barton, G.J., 2000. Application of multiple sequence alignment profiles to improve protein secondary structure prediction. Proteins Struct Funct Bioinform 40, 502511. https://doi.org/10.1002/1097-0134(20000815)40:3<502::aid-prot170>3.0.co;2-q

24. Dalbey, R.E., Kuhn, A., 2015. Membrane Insertases Are Present in All Three Domains of Life. Structure 23, 1559-1560. https://doi.org/10.1016/j.str.2015.08.002

25. Diamantopoulou, A., Sun, Z., Mukai, J., Xu, B., Fenelon, K., Karayiorgou, M., Gogos, J.A., 2017. Loss-of-function mutation inMirta22/Emc10rescues specific schizophreniarelated phenotypes in a mouse model of the 22q11.2 deletion. Proc National Acad Sci 114, E6127-E6136. https://doi.org/10.1073/pnas.1615719114

26. Dickinson, M.E., Flenniken, A.M., Ji, X., Teboul, L., Wong, M.D., White, J.K., Meehan, T.F., Weninger, W.J., Westerberg, H., Adissu, H., Baker, C.N., Bower, L., Brown, J.M., Caddle, L.B., Chiani, F., Clary, D., Cleak, J., Daly, M.J., Denegre, J.M., Doe, B., Dolan, M.E., Edie, S.M., Fuchs, H., Gailus-Durner, V., Galli, A., Gambadoro, A., Gallegos, J., Guo, S., Horner, N.R., Hsu, C.-W., Johnson, S.J., Kalaga, S., Keith, L.C., Lanoue, L., Lawson, T.N., Lek, M., Mark, M., Marschall, S., Mason, J., McElwee, M.L., 
Newbigging, S., Nutter, L.M.J., Peterson, K.A., Ramirez-Solis, R., Rowland, D.J., Ryder, E., Samocha, K.E., Seavitt, J.R., Selloum, M., Szoke-Kovacs, Z., Tamura, M., Trainor, A.G., Tudose, I., Wakana, S., Warren, J., Wendling, O., West, D.B., Wong, L., Yoshiki, A., McKay, M., Urban, B., Lund, C., Froeter, E., LaCasse, T., Mehalow, A., Gordon, E., Donahue, L.R., Taft, R., Kutney, P., Dion, S., Goodwin, L., Kales, S., Urban, R., Palmer, K., Pertuy, F., Bitz, D., Weber, B., Goetz-Reiner, P., Jacobs, H., Marchand, E.L., Amri, A.E., Fertak, L.E., Ennah, H., Ali-Hadji, D., Ayadi, A., Wattenhofer-Donze, M., Jacquot, S., André, P., Birling, M.-C., Pavlovic, G., Sorg, T., Morse, I., Benso, F., Stewart, M.E., Copley, C., Harrison, J., Joynson, S., Guo, R., Qu, D., Spring, S., Yu, L., Ellegood, J., Morikawa, L., Shang, X., Feugas, P., Creighton, A., Penton, P.C., Danisment, O., Griggs, N., Tudor, C.L., Green, A.L., Mazzeo, C.I., Siragher, E., Lillistone, C., Tuck, E., Gleeson, D., Sethi, D., Bayzetinova, T., Burvill, J., Habib, B., Weavers, L., Maswood, R., Miklejewska, E., Woods, M., Grau, E., Newman, S., Sinclair, C., Brown, E., Ayabe, S., Iwama, M., Murakami, A., Wurst, W., MacArthur, D.G., Tocchini-Valentini, G.P., Gao, X., Flicek, P., Bradley, A., Skarnes, W.C., Justice, M.J., Parkinson, H.E., Moore, M., Wells, S., Braun, R.E., Svenson, K.L., Angelis, M.H. de, Herault, Y., Mohun, T., Mallon, A.-M., Henkelman, R.M., Brown, S.D.M., Adams, D.J., Lloyd, K.C.K., McKerlie, C., Beaudet, A.L., Bućan, M., Murray, S.A., 2016. High-throughput discovery of novel developmental phenotypes. Nature 537, 508-514. https://doi.org/10.1038/nature19356 https://www.mousephenotype.org/publications/

27. Ellgaard, L., McCaul, N., Chatsisvili, A., Braakman, I., 2016. Co- and Post-Translational Protein Folding in the ER: Co- and Post-Translational Protein Folding in the ER. Traffic 17, 615-638. https://doi.org/10.1111/tra. 12392

28. Emsley, P., Cowtan, K., 2004. Coot: model-building tools for molecular graphics. Acta Crystallogr Sect D Biological Crystallogr 60, 2126-2132. https://doi.org/10.1107/s0907444904019158

29. Emsley, P., Lohkamp, B., Scott, W.G., Cowtan, K., 2010. Features and development of Coot. Acta Crystallogr Sect D Biological Crystallogr 66, 486-501. https://doi.org/10.1107/s0907444910007493

30. Goddard, T.D., Huang, C.C., Meng, E.C., Pettersen, E.F., Couch, G.S., Morris, J.H., Ferrin, T.E., 2018. UCSF ChimeraX: Meeting modern challenges in visualization and analysis. Protein Sci 27, 14-25. https://doi.org/10.1002/pro.3235

31. Goehring, A., Lee, C.-H., Wang, K.H., Michel, J.C., Claxton, D.P., Baconguis, I., Althoff, T., Fischer, S., Garcia, K.C., Gouaux, E., 2014. Screening and large-scale expression of membrane proteins in mammalian cells for structural studies. Nat Protoc 9, 2574-2585. https://doi.org/10.1038/nprot.2014.173

32. Graham, J.B., Canniff, N.P., Hebert, D.N., 2019. TPR-containing proteins control protein organization and homeostasis for the endoplasmic reticulum. Crit Rev Biochem Mol 54, 103-118. https://doi.org/10.1080/10409238.2019.1590305

33. Guna, A., Volkmar, N., Christianson, J.C., Hegde, R.S., 2017. The ER membrane protein complex is a transmembrane domain insertase. Science 359, 470-473. https://doi.org/10.1126/science.aao3099

34. Hagn, F., Nasr, M.L., Wagner, G., 2018. Assembly of phospholipid nanodiscs of controlled size for structural studies of membrane proteins by NMR. Nat Protoc 13, 79 98. https://doi.org/10.1038/nprot.2017.094

35. Halbleib, K., Pesek, K., Covino, R., Hofbauer, H.F., Wunnicke, D., Hänelt, I., Hummer, G., Ernst, R., 2017. Activation of the Unfolded Protein Response by Lipid Bilayer Stress. Mol Cell 67, 673-684.e8. https://doi.org/10.1016/j.molcel.2017.06.012

36. Harel, T., Yesil, G., Bayram, Y., Coban-Akdemir, Z., Charng, W.-L., Karaca, E., Asmari, A.A., Eldomery, M.K., Hunter, J.V., Jhangiani, S.N., Rosenfeld, J.A., Pehlivan, D., ElHattab, A.W., Saleh, M.A., LeDuc, C.A., Muzny, D., Boerwinkle, E., Genomics, B.-H.C. 
for M., Gibbs, R.A., Chung, W.K., Yang, Y., Belmont, J.W., Lupski, J.R., 2016. Monoallelic and Biallelic Variants in EMC1 Identified in Individuals with Global Developmental Delay, Hypotonia, Scoliosis, and Cerebellar Atrophy. Am J Hum Genet 98, 562-70. https://doi.org/10.1016/j.ajhg.2016.01.011

37. Heberle, F.A., Doktorova, M., Scott, H.L., Skinkle, A.D., Waxham, M.N., Levental, I., 2020. Direct label-free imaging of nanodomains in biomimetic and biological membranes by cryogenic electron microscopy. Proc National Acad Sci 202002200. https://doi.org/10.1073/pnas.2002200117

38. Hiramatsu, N., Tago, T., Satoh, T., Satoh, A.K., 2019. ER membrane protein complex is required for the insertions of late-synthesized transmembrane helices of Rh1 in Drosophila photoreceptors. Mol Biol Cell 30, 2890-2900. https://doi.org/10.1091/mbc.e19-08-0434

39. Hofbauer, H.F., Gecht, M., Fischer, S.C., Seybert, A., Frangakis, A.S., Stelzer, E.H.K., Covino, R., Hummer, G., Ernst, R., 2018. The molecular recognition of phosphatidic acid by an amphipathic helix in Opi1Headgroup selectivity of an amphipathic helix. J Cell Biology 217, 3109-3126. https://doi.org/10.1083/jcb.201802027

40. Hu, M., Yu, H., Gu, K., Wang, Z., Ruan, H., Wang, K., Ren, S., Li, B., Gan, L., Xu, S., Yang, G., Shen, Y., Li, X., 2018. A particle-filter framework for robust cryo-EM 3D reconstruction. Nat Methods 15, 1083-1089. https://doi.org/10.1038/s41592-018-0223-8

41. Jacquemyn, J., Cascalho, A., Goodchild, R.E., 2017. The ins and outs of endoplasmic reticulum-controlled lipid biosynthesis. Embo Rep 18, 1905-1921. https://doi.org/10.15252/embr.20164342

42. Jones, D.T., 1999. Protein secondary structure prediction based on position-specific scoring matrices. J Mol Biol 292, 195-202. https://doi.org/10.1006/jmbi.1999.3091

43. Jones, D.T., Taylor, W.R., Thornton, J.M., 1994. A Model Recognition Approach to the Prediction of All-Helical Membrane Protein Structure and Topology. Biochemistry-us 33, 3038-3049. https://doi.org/10.1021/bi00176a037

44. Jonikas, M.C., Collins, S.R., Denic, V., Oh, E., Quan, E.M., Schmid, V., Weibezahn, J., Schwappach, B., Walter, P., Weissman, J.S., Schuldiner, M., 2009. Comprehensive characterization of genes required for protein folding in the endoplasmic reticulum. Sci New York N Y 323, 1693-7. https://doi.org/10.1126/science.1167983

45. Junes-Gill, K.S., Gallaher, T.K., Gluzman-Poltorak, Z., Miller, J.D., Wheeler, C.J., Fan, X., Basile, L.A., 2010. hHSS1: a novel secreted factor and suppressor of glioma growth located at chromosome 19q13.33. J Neuro-oncol 102, 197-211. https://doi.org/10.1007/s11060-010-0314-6

46. Källberg, M., Wang, H., Wang, S., Peng, J., Wang, Z., Lu, H., Xu, J., 2012. Templatebased protein structure modeling using the RaptorX web server. Nat Protoc 7, 15111522. https://doi.org/10.1038/nprot.2012.085

47. Kelley, L.A., Mezulis, S., Yates, C.M., Wass, M.N., Sternberg, M.J.E., 2015. The Phyre2 web portal for protein modeling, prediction and analysis. Nat Protoc 10, 845-858. https://doi.org/10.1038/nprot.2015.053

48. Kim J, Stroud RM, Craik CS. Rapid identification of recombinant Fabs that bind to membrane proteins. Methods. 2011;55(4):303-309. doi:10.1016/j.ymeth.2011.09.012

49. Kimanius, D., Forsberg, B.O., Scheres, S.H., Lindahl, E., 2016. Accelerated cryo-EM structure determination with parallelisation using GPUs in RELION-2. Elife 5, e18722. https://doi.org/10.7554/elife.18722

50. Krogh, A., Larsson, B., Heijne, G. von, Sonnhammer, E.L.L., 2001. Predicting transmembrane protein topology with a hidden markov model: application to complete genomes. J Mol Biol 305, 567-580. https://doi.org/10.1006/jmbi.2000.4315

51. Krysztofinska, E.M., Evans, N.J., Thapaliya, A., Murray, J.W., Morgan, R.M.L., Martinez-Lumbreras, S., Isaacson, R.L., 2017. Structure and Interactions of the TPR 
Domain of Sgt2 with Yeast Chaperones and Ybr137wp. Frontiers Mol Biosci 4, 68. https://doi.org/10.3389/fmolb.2017.00068

52. Kudze, T., Mendez-Dorantes, C., Jalloh, C.S., McClellan, A.J., 2018. Evidence for interaction between Hsp90 and the ER membrane complex. Cell Stress Chaperones 23, 1101-1115. https://doi.org/10.1007/s12192-018-0908-z

53. Kumazaki, K., Kishimoto, T., Furukawa, A., Mori, H., Tanaka, Y., Dohmae, N., Ishitani, R., Tsukazaki, T., Nureki, O., 2014. Crystal structure of Escherichia coli YidC, a membrane protein chaperone and insertase. Sci Rep-uk 4, 7299. https://doi.org/10.1038/srep07299

54. Lahiri, S., Chao, J.T., Tavassoli, S., Wong, A.K.O., Choudhary, V., Young, B.P., Loewen, C.J.R., Prinz, W.A., 2014. A conserved endoplasmic reticulum membrane protein complex (EMC) facilitates phospholipid transfer from the ER to mitochondria. Plos Biol 12, e1001969. https://doi.org/10.1371/journal.pbio.1001969

55. Laverty, D., Desai, R., Uchański, T., Masiulis, S., Stec, W.J., Malinauskas, T., Zivanov, J., Pardon, E., Steyaert, J., Miller, K.W., Aricescu, A.R., 2019. Cryo-EM structure of the human $\alpha 1 \beta 3 \gamma 2$ GABAA receptor in a lipid bilayer. Nature 565, 516-520. https://doi.org/10.1038/s41586-018-0833-4

56. Lin, D.L., Inoue, T., Chen, Y.-J., Chang, A., Tsai, B., Tai, A.W., 2019. The ER Membrane Protein Complex Promotes Biogenesis of Dengue and Zika Virus Nonstructural Multi-pass Transmembrane Proteins to Support Infection. Cell Reports 27, 1666-1674.e4. https://doi.org/10.1016/j.celrep.2019.04.051

57. Louie, R.J., Guo, J., Rodgers, J.W., White, R., Shah, N.A., Pagant, S., Kim, P., Livstone, M., Dolinski, K., McKinney, B.A., Hong, J., Sorscher, E.J., Bryan, J., Miller, E.A., Hartman, J.L., 2012. A yeast phenomic model for the gene interaction network modulating CFTR- $\triangle$ F508 protein biogenesis. Genome Med 4, 103. https://doi.org/10.1186/gm404

58. Li, X., Mooney, P., Zheng, S., Booth, C.R., Braunfeld, M.B., Gubbens, S., Agard, D.A., Cheng, Y., 2013. Electron counting and beam-induced motion correction enable nearatomic-resolution single-particle cryo-EM. Nat Methods 10, 584-590. https://doi.org/10.1038/nmeth.2472

59. Liebschner, D., Afonine, P.V., Baker, M.L., Bunkóczi, G., Chen, V.B., Croll, T.I., Hintze, B., Hung, L.-W., Jain, S., McCoy, A.J., Moriarty, N.W., Oeffner, R.D., Poon, B.K., Prisant, M.G., Read, R.J., Richardson, J.S., Richardson, D.C., Sammito, M.D., Sobolev, O.V., Stockwell, D.H., Terwilliger, T.C., Urzhumtsev, A.G., Videau, L.L., Williams, C.J., Adams, P.D., 2019. Macromolecular structure determination using Xrays, neutrons and electrons: recent developments in Phenix. Acta Crystallogr Sect D 75, 861-877. https://doi.org/10.1107/s2059798319011471

60. Luo, W., Gong, X., Chang, A., 2002. An ER Membrane Protein, Sop4, Facilitates ER Export of the Yeast Plasma Membrane [H+]ATPase, Pma1. Traffic 3, 730-739. https://doi.org/10.1034/j.1600-0854.2002.31005.x

61. Lupas, A., Dyke, M.V., Stock, J., 1991. Predicting coiled coils from protein sequences. Science 252, 1162-1164. https://doi.org/10.1126/science.252.5009.1162

62. Marinko, J.T., Huang, H., Penn, W.D., Capra, J.A., Schlebach, J.P., Sanders, C.R., 2019. Folding and Misfolding of Human Membrane Proteins in Health and Disease: From Single Molecules to Cellular Proteostasis. Chem Rev 119, 5537-5606. https://doi.org/10.1021/acs.chemrev.8b00532

63. Marquez, J., Criscione, J., Charney, R.M., Prasad, M.S., Hwang, W.Y., Mis, E.K., García-Castro, M.I., Khokha, M.K., 2020. Disrupted ER membrane protein complexmediated topogenesis drives congenital neural crest defects. J Clin Invest 130, 813-826. https://doi.org/10.1172/jci129308 
64. Mastronarde, D.N., 2005. Automated electron microscope tomography using robust prediction of specimen movements. J Struct Biol 152, 36-51.

https://doi.org/10.1016/j.jsb.2005.07.007

65. Mitra, K., Ubarretxena-Belandia, I., Taguchi, T., Warren, G., Engelman, D.M., 2004. Modulation of the bilayer thickness of exocytic pathway membranes by membrane proteins rather than cholesterol. Proc National Acad Sci 101, 4083-4088. https://doi.org/10.1073/pnas.0307332101

66. Morin, A., Eisenbraun, B., Key, J., Sanschagrin, P.C., Timony, M.A., Ottaviano, M., Sliz, P., 2013. Collaboration gets the most out of software. Elife 2, e01456. https://doi.org/10.7554/elife.01456

67. Ngo, A.M., Shurtleff, M.J., Popova, K.D., Kulsuptrakul, J., Weissman, J.S., Puschnik, A.S., 2019. The ER membrane protein complex is required to ensure correct topology and stable expression of flavivirus polyproteins. Elife 8, e48469. https://doi.org/10.7554/elife.48469

68. Notredame, C., Higgins, D.G., Heringa, J., 2000. T-coffee: a novel method for fast and accurate multiple sequence alignment. J Mol Biol 302, 205-217. https://doi.org/10.1006/jmbi.2000.4042

69. O’Donnell, J.P., Phillips, B.P., Yagita, Y., Juszkiewicz, S., Wagner, A., Malinverni, D., Keenan, R.J., Miller, E.A., Hegde, R.S., 2020. The architecture of EMC reveals a path for membrane protein insertion. Elife 9, e57887. https://doi.org/10.7554/elife.57887

70. Obradovic, Z., Peng, K., Vucetic, S., Radivojac, P., Dunker, A.K., 2005. Exploiting heterogeneous sequence properties improves prediction of protein disorder. Proteins Struct Funct Bioinform 61, 176-182. https://doi.org/10.1002/prot.20735

71. Ouali, M., King, R.D., 2000. Cascaded multiple classifiers for secondary structure prediction. Protein Sci 9, 1162-1176. https://doi.org/10.1110/ps.9.6.1162

72. Peng, K., Radivojac, P., Vucetic, S., Dunker, A.K., Obradovic, Z., 2006. Lengthdependent prediction of protein intrinsic disorder. Bmc Bioinformatics 7, 208. https://doi.org/10.1186/1471-2105-7-208

73. Petkovic, M., Oses-Prieto, J., Burlingame, A., Jan, L.Y., Jan, Y.N., 2020. TMEM16K is an interorganelle regulator of endosomal sorting. Nat Commun 11, 3298. https://doi.org/10.1038/s41467-020-17016-8

74. Pettersen, E.F., Goddard, T.D., Huang, C.C., Couch, G.S., Greenblatt, D.M., Meng, E.C., Ferrin, T.E., 2004. UCSF Chimera-A visualization system for exploratory research and analysis. J Comput Chem 25, 1605-1612. https://doi.org/10.1002/jcc.20084

75. Pfeffer, S., Dudek, J., Schaffer, M., Ng, B.G., Albert, S., Plitzko, J.M., Baumeister, W., Zimmermann, R., Freeze, H.H., Engel, B.D., Förster, F., 2017. Dissecting the molecular organization of the translocon-associated protein complex. Nat Commun 8, 14516. https://doi.org/10.1038/ncomms 14516

76. Pleiner, T., Tomaleri, G.P., Januszyk, K., Inglis, A.J., Hazu, M., Voorhees, R.M., 2020. Structural basis for membrane insertion by the human ER membrane protein complex. Science eabb5008. https://doi.org/10.1126/science.abb5008

77. Probert, F., Rice, P., Scudamore, C.L., Wells, S., Williams, R., Hough, T.A., Cox, I.J., 2015. 1H NMR Metabolic Profiling of Plasma Reveals Additional Phenotypes in Knockout Mouse Models. J Proteome Res 14, 2036-2045. https://doi.org/10.1021/pr501039k

78. Punjani, A., Rubinstein, J.L., Fleet, D.J., Brubaker, M.A., 2017. cryoSPARC: algorithms for rapid unsupervised cryo-EM structure determination. Nat Methods 14, 290-296. https://doi.org/10.1038/nmeth.4169

79. Punjani, A., Zhang, H., Fleet, D.J., 2019. Non-uniform refinement: Adaptive regularization improves single particle cryo-EM reconstruction. Biorxiv 2019.12.15.877092. https://doi.org/10.1101/2019.12.15.877092 
80. Punjani, A., Fleet, D.J., 2020. 3D Variability Analysis: Directly resolving continuous flexibility and discrete heterogeneity from single particle cryo-EM images. Biorxiv 2020.04.08.032466. https://doi.org/10.1101/2020.04.08.032466

81. Raman, S., Vernon, R., Thompson, J., Tyka, M., Sadreyev, R., Pei, J., Kim, D., Kellogg, E., DiMaio, F., Lange, O., Kinch, L., Sheffler, W., Kim, B., Das, R., Grishin, N.V., Baker, D., 2009. Structure prediction for CASP8 with all-atom refinement using Rosetta. Proteins Struct Funct Bioinform 77, 89-99. https://doi.org/10.1002/prot.22540

82. Ramírez, A.S., Kowal, J., Locher, K.P., 2019. Cryo-electron microscopy structures of human oligosaccharyltransferase complexes OST-A and OST-B. Science 366, 13721375. https://doi.org/10.1126/science.aaz3505

83. Ramlaul, K., Palmer, C.M., Nakane, T., Aylett, C.H.S., 2020. Mitigating local overfitting during single particle reconstruction with SIDESPLITTER. J Struct Biol 211, 107545. https://doi.org/10.1016/j.jsb.2020.107545

84. Rappsilber, J., Mann, M., Ishihama, Y., 2007. Protocol for micro-purification, enrichment, pre-fractionation and storage of peptides for proteomics using StageTips. Nat Protoc 2, 1896-1906. https://doi.org/10.1038/nprot.2007.261

85. Reinisch, K.M., Camilli, P.D., 2015. SMP-domain proteins at membrane contact sites: Structure and function. Biochim Biophys Acta 1861, 924-7. https://doi.org/10.1016/j.bbalip.2015.12.003

86. Richard, M., Boulin, T., Robert, V.J.P., Richmond, J.E., Bessereau, J.-L., 2013. Biosynthesis of ionotropic acetylcholine receptors requires the evolutionarily conserved ER membrane complex. Proc National Acad Sci 110, E1055-E1063. https://doi.org/10.1073/pnas.1216154110

87. Ritchie, T.K., Grinkova, Y.V., Bayburt, T.H., Denisov, I.G., Zolnerciks, J.K., Atkins, W.M., Sligar, S.G., 2009. Chapter Eleven Reconstitution of Membrane Proteins in Phospholipid Bilayer Nanodiscs. Methods Enzymol 464, 211-231. https://doi.org/10.1016/s0076-6879(09)64011-8

88. Rost, B., 2001. Review: Protein Secondary Structure Prediction Continues to Rise. J Struct Biol 134, 204-218. https://doi.org/10.1006/jsbi.2001.4336

89. Rothstein, R., 1991. [19] Targeting, disruption, replacement, and allele rescue: Integrative DNA transformation in yeast. Methods Enzymol 194, 281-301. https://doi.org/10.1016/0076-6879(91)94022-5

90. Roy, A., Kucukural, A., Zhang, Y., 2010. I-TASSER: a unified platform for automated protein structure and function prediction. Nat Protoc 5, 725-738. https://doi.org/10.1038/nprot.2010.5

91. Salas-Estrada, L.A., Leioatts, N., Romo, T.D., Grossfield, A., 2018. Lipids Alter Rhodopsin Function via Ligand-like and Solvent-like Interactions. Biophys J 114, 355367. https://doi.org/10.1016/j.bpj.2017.11.021

92. Samuelson, J.C., Chen, M., Jiang, F., Möller, I., Wiedmann, M., Kuhn, A., Phillips, G.J., Dalbey, R.E., 2000. YidC mediates membrane protein insertion in bacteria. Nature 406, 637-641. https://doi.org/10.1038/35020586

93. Satoh, T., Ohba, A., Liu, Z., Inagaki, T., Satoh, A.K., 2015. dPob/EMC is essential for biosynthesis of rhodopsin and other multi-pass membrane proteins in Drosophila photoreceptors. Elife 4, e06306. https://doi.org/10.7554/elife.06306

94. Savidis, G., McDougall, W.M., Meraner, P., Perreira, J.M., Portmann, J.M., Trincucci, G., John, S.P., Aker, A.M., Renzette, N., Robbins, D.R., Guo, Z., Green, S., Kowalik, T.F., Brass, A.L., 2016. Identification of Zika Virus and Dengue Virus Dependency Factors using Functional Genomics. Cell Reports 16, 232-246. https://doi.org/10.1016/j.celrep.2016.06.028

95. Scheufler, C., Brinker, A., Bourenkov, G., Pegoraro, S., Moroder, L., Bartunik, H., Hartl, F.U., Moarefi, I., 2000. Structure of TPR Domain-Peptide Complexes Critical Elements 
in the Assembly of the Hsp70-Hsp90 Multichaperone Machine. Cell 101, 199-210. https://doi.org/10.1016/s0092-8674(00)80830-2

96. Schlegel, T., Mirus, O., Haeseler, A. von, Schleiff, E., 2007. The Tetratricopeptide Repeats of Receptors Involved in Protein Translocation across Membranes. Mol Biol Evol 24, 2763-2774. https://doi.org/10.1093/molbev/msm211

97. Schwanhäusser, B., Busse, D., Li, N., Dittmar, G., Schuchhardt, J., Wolf, J., Chen, W., Selbach, M., 2011. Global quantification of mammalian gene expression control. Nature 473, 337-342. https://doi.org/10.1038/nature 10098

98. Sejdiu, B.I., Tieleman, D.P., 2020. Lipid-Protein Interactions Are a Unique Property and Defining Feature of G Protein-Coupled Receptors. Biophys J 118, 1887-1900. https://doi.org/10.1016/j.bpj.2020.03.008

99. Shaikh, T.R., Gao, H., Baxter, W.T., Asturias, F.J., Boisset, N., Leith, A., Frank, J., 2008. SPIDER image processing for single-particle reconstruction of biological macromolecules from electron micrographs. Nat Protoc 3, 1941-1974. https://doi.org/10.1038/nprot.2008.156

100. Shurtleff, M.J., Itzhak, D.N., Hussmann, J.A., Oakdale, N.T.S., Costa, E.A., Jonikas, M., Weibezahn, J., Popova, K.D., Jan, C.H., Sinitcyn, P., Vembar, S.S., Hernandez, H., Cox, J., Burlingame, A.L., Brodsky, J.L., Frost, A., Borner, G.H., Weissman, J.S., 2018. The ER membrane protein complex interacts cotranslationally to enable biogenesis of multipass membrane proteins. Elife 7, e37018.

https://doi.org/10.7554/elife.37018

101. Song, Y., DiMaio, F., Wang, R.Y.-R., Kim, D., Miles, C., Brunette, T., Thompson, J., Baker, D., 2013. High-Resolution Comparative Modeling with RosettaCM. Structure 21, 1735-1742. https://doi.org/10.1016/j.str.2013.08.005

102. Stagg, S.M., Noble, A.J., Spilman, M., Chapman, M.S., 2014. ResLog plots as an empirical metric of the quality of cryo-EM reconstructions. J Struct Biol 185, 418-426. https://doi.org/10.1016/j.jsb.2013.12.010

103. Suloway, C.J.M., Chartron, J.W., Zaslaver, M., Clemons, W.M., 2009. Model for eukaryotic tail-anchored protein binding based on the structure of Get3. Proc National Acad Sci 106, 14849-14854. https://doi.org/10.1073/pnas.0907522106

104. Talbot, B.E., Vandorpe, D.H., Stotter, B.R., Alper, S.L., Schlondorff, J.S., 2019. Transmembrane insertases and N-glycosylation critically determine synthesis, trafficking, and activity of the nonselective cation channel TRPC6. J Biol Chem 294, 12655-12669. https://doi.org/10.1074/jbc.ra119.008299

105. Tang, X., Snowball, J.M., Xu, Y., Na, C.-L., Weaver, T.E., Clair, G., Kyle, J.E., Zink, E.M., Ansong, C., Wei, W., Huang, M., Lin, X., Whitsett, J.A., 2017. EMC3 coordinates surfactant protein and lipid homeostasis required for respiration. J Clin Invest 127, 4314-4325. https://doi.org/10.1172/jci94152

106. Tian, S., Wu, Q., Zhou, B., Choi, M.Y., Ding, B., Yang, W., Dong, M., 2019. Proteomic Analysis Identifies Membrane Proteins Dependent on the ER Membrane Protein Complex. Cell Reports 28, 2517-2526.e5.

https://doi.org/10.1016/j.celrep.2019.08.006

107. Volkmar, N., Thezenas, M.-L., Louie, S.M., Juszkiewicz, S., Nomura, D.K., Hegde, R.S., Kessler, B.M., Christianson, J.C., 2018. The ER membrane protein complex promotes biogenesis of sterol-related enzymes maintaining cholesterol homeostasis. J Cell Sci 132, jcs223453. https://doi.org/10.1242/jcs.223453

108. Volkmar, N., Christianson, J.C., 2020. Squaring the EMC - how promoting membrane protein biogenesis impacts cellular functions and organismal homeostasis. $\mathrm{J}$ Cell Sci 133, jcs243519. https://doi.org/10.1242/jcs.243519

109. Volmer, R., Ploeg, K. van der, Ron, D., 2013. Membrane lipid saturation activates endoplasmic reticulum unfolded protein response transducers through their 
transmembrane domains. Proc National Acad Sci 110, 4628-4633.

https://doi.org/10.1073/pnas.1217611110

110. Vyas, N.K., Vyas, M.N., Chervenak, M.C., Johnson, M.A., Pinto, B.M., Bundle, D.R., Quiocho, F.A., 2002. Molecular Recognition of Oligosaccharide Epitopes by a Monoclonal Fab Specific for Shigella flexneri Y Lipopolysaccharide: X-ray Structures and Thermodynamics. Biochemistry-us 41, 13575-13586.

https://doi.org/10.1021/bi0261387

111. Ward, J.J., Sodhi, J.S., McGuffin, L.J., Buxton, B.F., Jones, D.T., 2004.

Prediction and Functional Analysis of Native Disorder in Proteins from the Three Kingdoms of Life. J Mol Biol 337, 635-645. https://doi.org/10.1016/j.jmb.2004.02.002

112. Wainberg, M., Kamber, R.A., Balsubramani, A., Meyers, R.M., SinnottArmstrong, N., Hornburg, D., Jiang, L., Chan, J., Jian, R., Gu, M., Shcherbina, A., Dubreuil, M.M., Spees, K., Snyder, M.P., Kundaje, A., Bassik, M.C., 2019. A genomewide almanac of co-essential modules assigns function to uncharacterized genes. Biorxiv 827071. https://doi.org/10.1101/827071

113. Waterhouse, A.M., Procter, J.B., Martin, D.M.A., Clamp, M., Barton, G.J., 2009. Jalview Version 2-a multiple sequence alignment editor and analysis workbench.

Bioinformatics 25, 1189-1191. https://doi.org/10.1093/bioinformatics/btp033

114. Weissmann, F., Petzold, G., VanderLinden, R., Veld, P.J.H. in 't, Brown, N.G., Lampert, F., Westermann, S., Stark, H., Schulman, B.A., Peters, J.-M., 2016. biGBac enables rapid gene assembly for the expression of large multisubunit protein complexes. Proc National Acad Sci 113, E2564-E2569. https://doi.org/10.1073/pnas.1604935113

115. Wideman, J.G., 2015. The ubiquitous and ancient ER membrane protein complex (EMC): tether or not? F1000research 4, 624.

https://doi.org/10.12688/f1000research.6944.1

116. Wu, S., Avila-Sakar, A., Kim, J., Booth, D.S., Greenberg, C.H., Rossi, A., Liao, M., Li, X., Alian, A., Griner, S.L., Juge, N., Yu, Y., Mergel, C.M., Chaparro-Riggers, J., Strop, P., Tampé, R., Edwards, R.H., Stroud, R.M., Craik, C.S., Cheng, Y., 2012. Fabs Enable Single Particle cryoEM Studies of Small Proteins. Structure 20, 582-592. https://doi.org/10.1016/j.str.2012.02.017

117. Xiong, L., Zhang, L., Yang, Y., Li, N., Lai, W., Wang, F., Zhu, X., Wang, T., 2020. ER complex proteins are required for rhodopsin biosynthesis and photoreceptor survival in Drosophila and mice. Cell Death Differ 27, 646-661.

https://doi.org/10.1038/s41418-019-0378-6

118. Yang, J., Yan, R., Roy, A., Xu, D., Poisson, J., Zhang, Y., 2015. The I-TASSER Suite: protein structure and function prediction. Nat Methods $12,7-8$.

https://doi.org/10.1038/nmeth.3213

119. Zhang, K., 2016. Gctf: Real-time CTF determination and correction. J Struct Biol 193, 1-12. https://doi.org/10.1016/j.jsb.2015.11.003

120. Zhang, S., Xu, C., Larrimore, K.E., Ng, D.T.W., 2017. Slp1-Emp65: A Guardian Factor that Protects Folding Polypeptides from Promiscuous Degradation. Cell 171, 346357.e12. https://doi.org/10.1016/j.cell.2017.08.036

121. Zhang, Y., 2008. I-TASSER server for protein 3D structure prediction. Bmc Bioinformatics 9, 40. https://doi.org/10.1186/1471-2105-9-40

122. Zheng, S.Q., Palovcak, E., Armache, J.-P., Verba, K.A., Cheng, Y., Agard, D.A., 2017. MotionCor2: anisotropic correction of beam-induced motion for improved cryoelectron microscopy. Nat Methods 14, 331-332. https://doi.org/10.1038/nmeth.4193

123. Zhou, Y., Wu, F., Zhang, M., Xiong, Z., Yin, Q., Ru, Y., Shi, H., Li, J., Mao, S., Li, Y., Cao, X., Hu, R., Liew, C.W., Ding, Q., Wang, X., Zhang, Y., 2018. EMC10 governs male fertility via maintaining sperm ion balance. J Mol Cell Biol 10, 503-514. https://doi.org/10.1093/jmcb/mjy024 
124. Zimmermann, L., Stephens, A., Nam, S.-Z., Rau, D., Kübler, J., Lozajic, M., Gabler, F., Söding, J., Lupas, A.N., Alva, V., 2017. A Completely Reimplemented MPI Bioinformatics Toolkit with a New HHpred Server at its Core. J Mol Biol 430, 2237 2243. https://doi.org/10.1016/j.jmb.2017.12.007

125. Zivanov, J., Nakane, T., Forsberg, B.O., Kimanius, D., Hagen, W.J., Lindahl, E., Scheres, S.H., 2018. New tools for automated high-resolution cryo-EM structure determination in RELION-3. Elife 7, e42166. https://doi.org/10.7554/elife.42166 